\title{
\begin{tabular}{l|l} 
Mibraries & DSpace@MIT
\end{tabular}
}

\author{
MIT Open Access Articles
}

\section{It takes three to tango: 2. Bubble dynamics in basaltic volcanoes and ramifications for modeling normal Strombolian activity}

The MIT Faculty has made this article openly available. Please share how this access benefits you. Your story matters.

Citation: Suckale, J., B. H. Hager, L. T. Elkins-Tanton, and J.-C. Nave (2010), It takes three to tango: 2. Bubble dynamics in basaltic volcanoes and ramifications for modeling normal Strombolian activity, J. Geophys. Res., 115, B07410, doi:10.1029/2009JB006917. (c) 2010. American Geophysical Union.

As Published: http://dx.doi.org/10.1029/2009jb006917

Publisher: American Geophysical Union

Persistent URL: http://hdl.handle.net/1721.1/61579

Version: Final published version: final published article, as it appeared in a journal, conference proceedings, or other formally published context

Terms of Use: Article is made available in accordance with the publisher's policy and may be subject to US copyright law. Please refer to the publisher's site for terms of use. 


\title{
It takes three to tango:
}

\section{Bubble dynamics in basaltic volcanoes and ramifications for modeling normal Strombolian activity}

\author{
Jenny Suckale, ${ }^{1}$ Bradford H. Hager, ${ }^{1}$ Linda T. Elkins-Tanton, ${ }^{1}$ and Jean-Christophe Nave ${ }^{2}$ \\ Received 2 September 2009; revised 21 February 2010; accepted 28 April 2010; published 20 July 2010.
}

[1] This is the second paper of two that examine numerical simulations of buoyancy-

driven flow in the presence of large viscosity contrasts. In the first paper, we demonstrated that a combination of three numerical tools, an extended ghost fluid type method, the level set approach, and the extension velocity technique, accurately simulates complex interface dynamics in the presence of large viscosity contrasts. In this paper, we use this threefold numerical method to investigate bubble dynamics in the conduits of basaltic volcanos with a focus on normal Strombolian eruptions. Strombolian type activity, named after the famously episodic eruptions at Stromboli volcano, is characterized by temporally discrete fountains of incandescent clasts. The mildly explosive nature of normal Strombolian activity, as compared to more effusive variants of basaltic volcanism, is related to the presence of dissolved gas in the magma, yielding a complex two-phase flow problem. We present a detailed scaling analysis allowing identification of the pertinent regime for a given flow problem. The dynamic interactions between gas and magma can be classified into three nondimensional regimes on the basis of bubble sizes and magma viscosity. Resolving the fluid dynamics at the scale of individual bubbles is not equally important in all three regimes: As long as bubbles remain small enough to be spherical, their dynamic interactions are limited compared to the rich spectrum of coalescence and breakup processes observed for deformable bubbles, in particular, once inertia ceases to be negligible. One key finding in our simulations is that both large gas bubbles and large conduit-filling gas pockets ("slugs") are prone to dynamic instabilities that lead to their rapid breakup during buoyancy-driven ascent. We provide upper bound estimates for the maximum stable bubble size in a given magmatic system and discuss the ramifications of our results for two commonly used models of normal Strombolian type activity, the rise-speed-dependent model and the collapsing foam model.

Citation: Suckale, J., B. H. Hager, L. T. Elkins-Tanton, and J.-C. Nave (2010), It takes three to tango: 2. Bubble dynamics in basaltic volcanoes and ramifications for modeling normal Strombolian activity, J. Geophys. Res., 115, B07410, doi:10.1029/2009JB006917.

\section{Introduction}

[2] Many basaltic volcanoes erupt substantial amounts of gas while expelling comparatively little degassed magma. This observation suggests that gas segregation must have occurred prior to eruption and warrants attributing the existence of explosive basaltic volcanism to the presence of exsolved volatiles. Since the exsolution of gas is largely suppressed in the case of sufficiently deep seafloor volcanism, explosive basaltic activity should be very rare at mid-ocean ridges [Head and Wilson, 2003] and expected to occur primarily at subaerial volcanoes, in agreement with observations.

\footnotetext{
${ }^{1}$ Department of Earth, Atmospheric, and Planetary Sciences, Massachusetts Institute of Technology, Cambridge, Massachusetts, USA.

${ }^{2}$ Department of Mathematics, Massachusetts Institute of Technology, Cambridge, Massachusetts, USA.

Copyright 2010 by the American Geophysical Union. 0148-0227/10/2009JB006917
}

[3] Substantial progress has been made in understanding the early stages of eruptions in basaltic volcanos, i.e., bubble nucleation and growth [e.g., Sparks, 1978; Mangan et al., 1993; Hurwitz and Navon, 1994; Toramaru, 1995; Gardner et al., 1999; Mourtada-Bonnefoi and Laporte, 2002; Proussevitch and Sahagian, 2005], and its late stages, i.e., fragmentation [e.g., Sparks et al., 1994; Mangan and Cashman, 1996; Papale, 1999; Zhang, 1999; Spieler et al., 2004; Namiki and Manga, 2008]. However, the complex fluid dynamical interactions that may arise between these two stages still pose many open questions [Gonnermann and Manga, 2007].

[4] This paper complements prior studies [Manga and Stone, 1993, 1994, 1995] by not only examining two-phase flow in the Stokes, but also in the Navier-Stokes regime at low to moderate Reynolds number $(R e)$. Analyzing flow behavior at finite $R e$ is of interest for Strombolian type eruptions because inertia is not generally negligible. Inertial effects become important because of (1) the low viscosity of basaltic magma as compared to more silicic counterparts and 
(2) the high rise speed of gas pockets in the meter range, which are thought to cause normal Strombolian activity [Blackburn et al., 1976; Vergniolle and Brandeis, 1994; Vergniolle et al., 1996; Harris and Ripepe, 2007].

[5] Refining our understanding of bubble dynamics in basaltic flow is important to (1) inform the interpretation of vesicle morphology and bubble size distributions observed in thin sections and the conclusions drawn for the flow conditions at quenching, (2) identify meaningful assumptions about interface dynamics used in two-phase flow models, which refrain from resolving the gas-magma interface directly, and (3) verify how the gasdynamics observed in laboratory experiments change at scales representative of volcanic systems. We devote special attention to identifying the ramifications of our results for the two commonly used models of explosive basaltic volcanism, the rise-speeddependent (RSD) model pioneered by Wilson [1980] and the collapsing foam (CF) model [Vergniolle and Jaupart, 1986; Jaupart and Vergniolle, 1988, 1989].

[6] Both models agree in relating normal Strombolian style eruptions to the presence of a segregated gas phase. More precisely, both models assume that normal Strombolian activity is caused by the bursting of large gas slugs at the free surface, an idea pioneered by Blackburn et al. [1976] and refined in a large number of subsequent studies. They differ, however, in the presumed location where this gas slug is thought to form. The CF model assumes that gas accumulation occurs in the magma chamber or, more generally, under a large roof area in the conduit, implying that the gas slug originates at moderate to great depth and ascends stably through the volcanic conduit. In contrast, the RSD model asserts that the gas slug forms gradually during ascent through a cascade of coalescence, implying that slug formation occurs at comparatively shallow depth. To compare these contrasting views and to verify the regimes for which they are applicable, we investigate the following two questions numerically: (1) How likely is a coalescence cascade, as suggested by the RSD model, and under which conditions could interactions between multiple bubbles lead to coalescence and formation of a single large gas slug? (2) How likely is it that large pockets of gas can ascend stably over long distances, as suggested by the CF model, and under which circumstances is that to be expected?

[7] This paper is structured in the following way: Section 2 introduces the governing equations and identifies the relevant nondimensional regimes. In section 3 we discuss theoretical constraints on bubble stability and estimate the maximum stable bubble sizes for typical basaltic systems. Section 4 describes our modeling approach and the details of the simulations we perform. Section 5 presents our results, and section 6 presents the ramifications of our findings for the applicability of the RSD and CF model. We end with a brief summary (section 7) of the main contributions of this paper to understanding explosive basaltic volcanism.

\section{Governing Equations and Scaling Analysis}

[8] The flow in each domain (i.e., gas and magma) satisfies the Navier-Stokes equation:

$$
\rho\left(\frac{\partial \mathbf{v}}{\partial t}+(\mathbf{v} \cdot \nabla) \mathbf{v}\right)=-\nabla p+\nabla \cdot\left[\mu\left(\nabla \mathbf{v}+(\nabla \mathbf{v})^{\mathrm{T}}\right)\right]+\rho \mathbf{g}
$$

and the incompressibility constraint

$$
\nabla \cdot \mathbf{v}=0 .
$$

Throughout the paper we denote the velocity field by $\mathbf{v}=$ $(u, v, w)^{\mathrm{T}}$, the pressure by $p$, the density by $\rho$, the dynamic viscosity by $\mu$, and the gravitational acceleration by $\mathbf{g}$. The boundary conditions at the gas-magma interface are a discontinuous step in both density

$$
\rho(\phi)= \begin{cases}\rho_{g} & \text { for } \phi<0 \\ \rho_{f} & \text { for } \phi \geq 0,\end{cases}
$$

and viscosity

$$
\mu(\phi)= \begin{cases}\mu_{g} & \text { for } \phi<0 \\ \mu_{f} & \text { for } \phi \geq 0\end{cases}
$$

where the subscripts refer to either "gas" or "fluid" and $\phi$ is the level set function used to represent the interface [see Suckale et al., 2010].

[9] Additionally, the jump condition at the interface needs to be fulfilled:

$$
\left[\left(\begin{array}{l}
\mathbf{n} \\
\mathbf{t}_{1} \\
\mathbf{t}_{2}
\end{array}\right)(p I-\tau) \mathbf{n}^{T}\right]=\left(\begin{array}{c}
\sigma \kappa \\
0 \\
0
\end{array}\right),
$$

where $I$ is the unit matrix, $\sigma$ is the surface tension, $\tau$ is the stress tensor, $\kappa$ is the curvature of the interface, and $\mathbf{n}, \mathbf{t}_{1}, \mathbf{t}_{2}$ are the normal vector and the two tangential vectors to the interface moving with the local fluid velocity.

[10] Using the sign of the level set function $\phi$ as an indicator for the two different phases in the manner defined above (equations (3) and (4)), we combine the governing equations for the two phases into one:

$$
\begin{aligned}
\rho(\phi)\left(\frac{\partial \mathbf{v}}{\partial t}+(\mathbf{v} \cdot \nabla) \mathbf{v}\right)= & -\nabla p+\nabla \cdot\left[\mu(\phi)\left(\nabla \mathbf{v}+(\nabla \mathbf{v})^{\mathrm{T}}\right)\right] \\
& +\mathbf{g} \rho(\phi)-\sigma \kappa(\phi) \delta(\phi) \mathbf{n}
\end{aligned}
$$

where the delta function $\delta(\phi)$ implies that surface tension only acts at the interface.

[11] The two-phase flow systems investigated in this paper span a wide range of fluid dynamical regimes. In order to nondimensionalize the governing equation (equation (6)), we define the following set of characteristic scales:

$$
\mathbf{x}=a \mathbf{x}^{\prime}, \quad \mathbf{v}=v_{0} \mathbf{v}^{\prime}, \quad t=\frac{a}{v_{0}} t^{\prime}, \quad p=v_{0} 2 \rho p^{\prime}, \quad \rho=\rho \rho^{\prime}, \quad \mu=\mu_{f} \mu^{\prime},
$$

where $v_{0}$ denotes the rise speed of the bubble or gas pocket, $a$ denotes the radius of the bubble, and $\mu_{f}$ denotes the viscosity of the magma. Substituting these characteristic quantities into equation (6), rearranging, and dropping the primes yields the nondimensional multiphase Navier-Stokes equation:

$$
\begin{aligned}
\rho(\phi)\left(\frac{\partial \mathbf{v}}{\partial t}+(\mathbf{v} \cdot \nabla) \mathbf{v}\right)= & -\nabla p+\frac{1}{R e} \nabla \cdot\left[\mu(\phi)\left(\nabla \mathbf{v}+(\nabla \mathbf{v})^{\mathrm{T}}\right)\right] \\
& +\frac{\rho(\phi)}{F r} \hat{\mathbf{z}}-\frac{1}{W e} \kappa(\phi) \delta(\phi) \mathbf{n},
\end{aligned}
$$




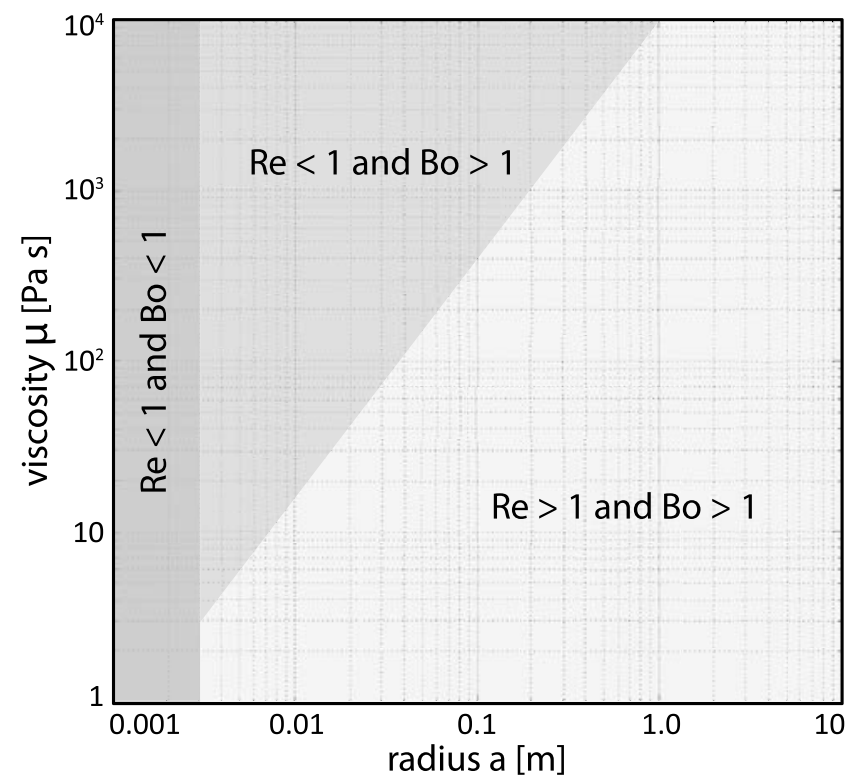

Figure 1. Dependence of the three main nondimensional domains on bubble radius and magma viscosity. We assume a magma density of $\rho_{f}=3500 \mathrm{~kg} / \mathrm{m}^{3}$ and a surface tension coefficient of $\sigma=0.3 \mathrm{~N} / \mathrm{m}$. The boundary of regime 1, characterized by spherical bubbles and negligible inertia, is determined only by the size of the bubbles because $B o$ does not depend on magma viscosity (see equation (13)). Regime 2, in which bubbles become deformable but inertia remains negligible, only exists at sufficiently high viscosity, $\mu \geq 3 \mathrm{~Pa} \mathrm{~s}$.

where $\hat{\mathbf{z}}$ denotes the unit vector in vertical direction. The boundary and jump conditions are nondimensionalized accordingly.

[12] Equation (8) highlights that the relevant nondimensional numbers are

Reynolds number

$$
R e=\frac{\rho_{f} v_{0} a}{\mu_{f}},
$$

Froude number

$$
F r=\frac{v_{0}^{2}}{g a},
$$

Weber number

$$
W e=\frac{\rho_{f} a v_{0}^{2}}{\sigma},
$$

Viscosity ratio

$$
\Pi_{1}=\frac{\mu_{f}}{\mu_{g}} .
$$

In the special case where $R e=0$, the nondimensional groups determining the dynamics of the system reduce to Bond or Eötvös number

$$
B o=\frac{\Delta \rho g a^{2}}{\sigma},
$$

and $\Pi_{1}$ as defined previously. Throughout the paper we use the variable $\Pi$ to denote nondimensional numbers that do not bear specific names. In section 4 , we introduce additional nondimensional ratios to characterize the initial condition, the relative position, and the size ratio of two bubbles.

[13] The nondimensional numbers (equations (9), (10), (11), and (13)) have intuitive physical interpretations: $R e$ is a measure of the relative importance of inertia to viscous forces. $F r$ is the ratio of inertial and buoyancy forces, and $W e$ is the ratio of inertia to surface tension. $B o$ is used to quantify the degree to which bubbles are able to deform in the Stokes regime. It corresponds to the ratio of viscous to surface tension forces, such that $B o \gg 1$ describes the case in which surface tension becomes largely negligible, resulting in extreme bubble deformability. In the special case of buoyancy-driven flow, the Capillary number $\mathrm{Ca}$ is identical to $B o$, which can be seen when substituting the Stokes rise speed into the expression for $C a$, yielding $C a=\mu v /$ $\sigma=\Delta \rho g a^{2} / \sigma=B o$.

[14] A wide range of flow regimes may arise in basaltic volcanism, because both the bubble radius and the viscosity of basaltic magma may vary by several orders of magnitude. The three main nondimensional regimes are illustrated in Figure 1. They are characterized by gas bubbles that are (1) spherical with negligible inertia $(B o<1$ and $R e<1)$, (2) deformable with negligible inertia $(B o>1$ and $R e<1)$, and (3) deformable with nonnegligible inertia $(B o>1$ and $R e>1)$. In the interest of completeness, we note that a nondimensional regime defined by $B o>1$ and $R e<1$ is conceivable theoretically but of little relevance in a geophysical context because it would imply an unrealistically low magma viscosity.

[15] All simulations presented in this paper were computed on a Cartesian grid using finite differences. The grid resolution of the computations is included in the respective figure captions and corresponds to the number of grid cells in the $\mathrm{x}, \mathrm{y}$, and $\mathrm{z}$ directions, if applicable. Detailed convergence tests are included in the auxiliary material. ${ }^{1}$ In the Stokes regime, we employ our recently developed method for resolving buoyancy-driven interface dynamics in the presence of large viscosity contrasts described in detail by Suckale et al. [2010]. Our Navier-Stokes solver is based on the projection method pioneered by Chorin [1968], combined with a ghost fluid based representation of the material discontinuities and jump conditions [Kang et al., 2000; Liu et al., 2000]. An abbreviated description of the discretization scheme can be found in the auxiliary material.

\section{Theoretical Estimates of Bubble Breakup}

[16] Theoretically, one would expect that the stable size of gas bubbles in stagnant fluids should be limited, because the stabilizing effect of surface tension on the bubble surface decreases rapidly with increasing bubble radius and because the large density contrast between gas and magma will cause perturbations to grow by a Rayleigh-Taylor instability. The latter tendency is counteracted by viscous forces slowing the rate of growth of unstable interfacial waves [e.g., Bellman and Pennington, 1954; Grace et al., 1978].

\footnotetext{
${ }^{1}$ Auxiliary materials are available in the HTML. doi:10.1029/ 2009JB006917.
} 


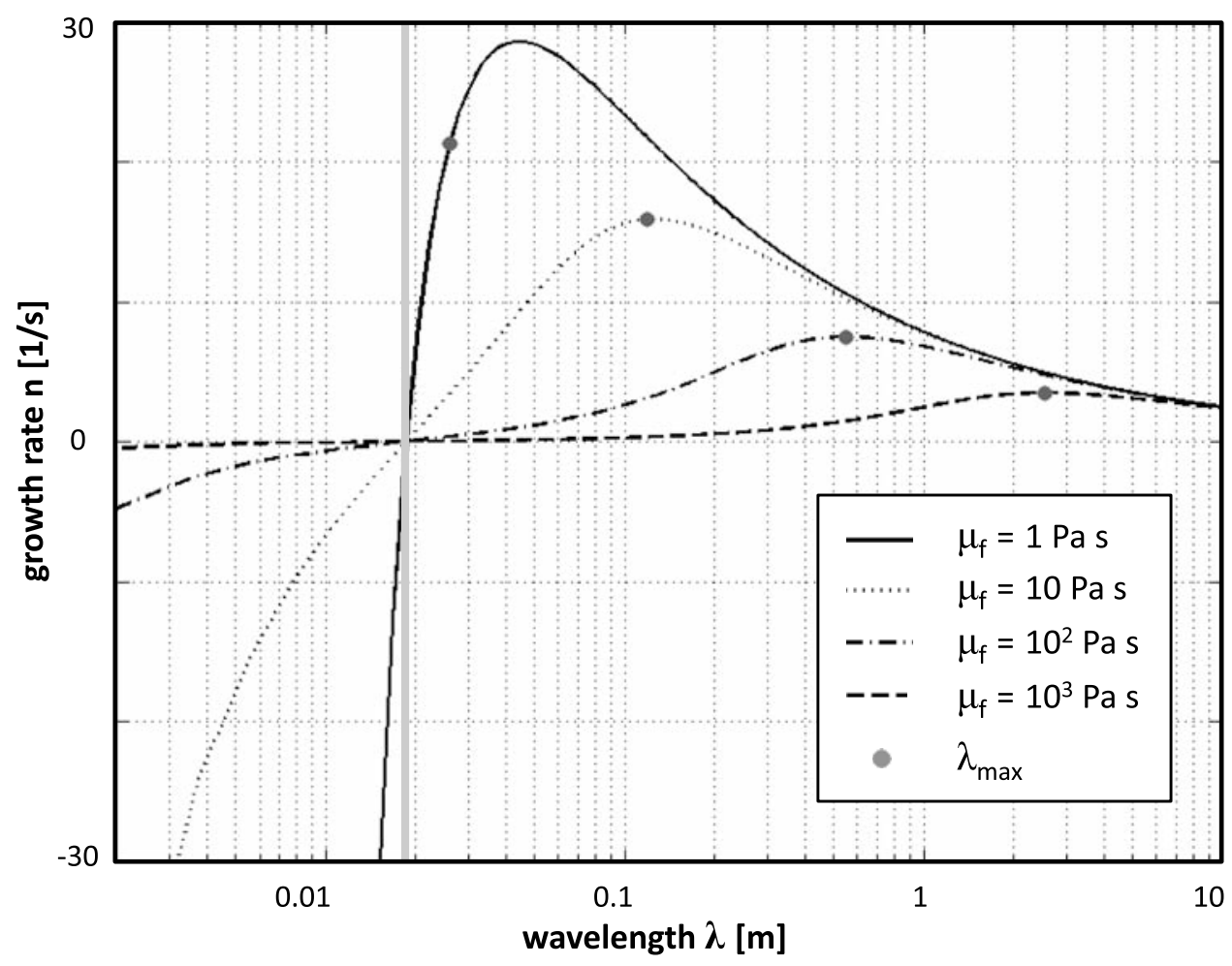

Figure 2. Wavelength dependence of the growth rate $n$ over a wide range of magma viscosities $\mu_{f}=$ $1,10,100$, and $1000 \mathrm{~Pa} \mathrm{~s}$. The other parameters used in the computation are $\rho_{f}=3500 \mathrm{~kg} / \mathrm{m}^{3}, \rho_{g}=$ $1.226 \mathrm{~kg} / \mathrm{m}^{3}, g=9.81 \mathrm{~m} / \mathrm{s}^{2}$, and $\sigma=0.3 \mathrm{~N} / \mathrm{m}$. The vertical gray line delimits the stable size range $a<\lambda_{\mathrm{cr}}$. The gray dots indicate the fastest growing wavelength for a specific $\mu_{f}$ from the approximative expression for $\lambda_{\max }$ (equation (19)) that is only valid at large viscosities.

[17] A disturbance of wavelength $\lambda$ on the bubble surface will be suppressed by surface tension if $\lambda$ is smaller than the critical value

$$
\lambda_{c r}=2 \pi \sqrt{\frac{\sigma}{g \Delta \rho}} .
$$

Thus, $\lambda_{c r}$ provides a lower bound for identifying potentially unstable wavelengths. A reasonable upper bound is half the circumference of the fluid particle

$$
\lambda_{u p}=\pi a,
$$

because a surface perturbation cannot exceed the bubble itself in size [Grace et al., 1978]. Together, $\lambda_{c r}$ and $\lambda_{u p}$ yield an estimate of the maximum stable bubble radius

$$
a_{c r}=2 \sqrt{\frac{\sigma}{g \Delta \rho}},
$$

implying that gas bubbles with radii in the millimeter range or larger $\left(a>a_{c r}\right)$ would be prone to breakup in basaltic liquid if the effect of viscosity was neglected.

[18] For small accelerations, Rayleigh-Taylor instabilities form only when a more dense fluid overlies a lighter one. Hence, it is the leading surface of the bubble where the first indentation of wavelength $\lambda>\lambda_{c r}$ forms. Splitting of the bubble, however, requires that this indentation grows fast enough to reach the trailing surface of the bubble before it is swept around to the equator of the bubble and stops growing through a Rayleigh-Taylor instability [Clift et al., 1974; Grace et al., 1978]. Growth of a disturbance occurs in several stages. During the early phase, while the amplitude of a disturbance is small with respect to its wavelength $\lambda$, growth is exponential and characterized by growth rate $n$. We assume that breakup only occurs if the instability reaches the base of the bubble during its exponential growth period. It follows that the maximum stable bubble size can be estimated by requiring that the time available for growth, $t_{a}$, exceeds that required for growth, $t_{g}$ [Grace et al., 1978].

[19] The time available for growth, $t_{a}$, depends on how fast a Rayleigh-Taylor instability in the center of the leading bubble surface is swept outward by viscous forces. The tangential velocity of the perturbation is determined by the terminal velocity of the bubble [Grace et al., 1978] yielding the approximate expression

$$
t_{a} \approx \frac{a}{v_{0}} \ln \left(\cot \left(\frac{\lambda}{8 a}\right)\right)
$$

in the limit of $\mu_{f} \gg \mu_{g}$ and for $\lambda_{c r}<\lambda<\lambda_{u p}$. We estimate the terminal velocity in equation (17) based on the empirical correlations summarized by Grace et al. [1978].

[20] The time required for growth, $t_{g}=1 / n$, depends on viscous damping of the interfacial waves and may be estimated, to a first approximation, from linearized stability theory [e.g., Bellman and Pennington, 1954]. For magmatic 
Table 1. Theoretical Prediction of the Maximum Stable Bubble Radius $a_{\max }$ in Basaltic Magma of Different Viscosities $\mu_{f}^{\mathrm{a}}$

\begin{tabular}{cc}
\hline $\begin{array}{c}\text { Magma Viscosity } \\
\mu_{f}(\mathrm{~Pa} \mathrm{~s})\end{array}$ & $\begin{array}{c}\text { Maximum Bubble Radius } \\
a_{\max }(\mathrm{m})\end{array}$ \\
\hline 10 & 0.06 \\
25 & 0.12 \\
50 & 0.18 \\
75 & 0.24 \\
100 & 0.29 \\
250 & 0.53 \\
500 & 0.84 \\
750 & 1.10 \\
1000 & 1.33 \\
\hline
\end{tabular}

additional parameters used in the computation: $\rho_{f}=3500 \mathrm{~kg} / \mathrm{m}^{3}, \rho_{g}=$ $1.226 \mathrm{~kg} / \mathrm{m}^{3}, g=9.81 \mathrm{~m} / \mathrm{s}^{2}$, surface tension $\sigma=0.3 \mathrm{~N} / \mathrm{m}$, and viscosity ratio $10^{-6}$.

systems where $\mu_{f} \gg \mu_{g}$, we can use the simplified expression [Plesset and Whipple, 1974]

$$
n^{2}+2 \frac{\mu_{f}}{\rho_{f}} k^{2} n-\frac{\Delta \rho}{\rho_{f}+\rho_{g}} g k+\frac{\sigma k^{3}}{\rho_{f}+\rho_{g}}=0
$$

where $k=2 \pi / \lambda$ is the wave number. Figure 2 summarizes the numerical solutions of equation (18) over several orders of magnitude in $\mu_{f}$. The dashed vertical line delimits the stable size regime where $a<\lambda_{c r}$. The gray dots indicate the surface wavelength which is expected to grow fastest for a given magma viscosity based on the approximative approach by Plesset and Whipple [1974]

$$
\lambda_{\max }=4 \pi\left(\frac{\mu_{f}^{2}}{\rho_{f}^{2} g} \frac{\rho_{f}+\rho_{g}}{\rho_{f}-\rho_{g}}\right)^{1 / 3} .
$$

[21] Grace et al. [1978] compared experimentally observed and theoretically predicted breakup times and concluded that gas bubbles rising through stagnant fluid break up if $t_{a}>$ $3.8 t_{g}$. Although their analysis was performed at much lower viscosities, we adopt this empirical criterion to estimate the maximum stable bubble radius for a wider range of magma viscosities (see Table 1). Given the semiempirical nature of the model and the simplifications it is based on [Batchelor, 1987], the values in Table 1 should be treated with some caution. For a detailed comparison of theoretical and computed bubble sizes, see section 5 .

\section{Simulations}

\subsection{Modeling Approach}

[22] The main objective of our study is to analyze bubble dynamics in basaltic conduits in which flow is driven primarily by gas. Since bubble ascent is only dynamically important if the bubble rise speed is large relative to the characteristic flow speed in the surrounding fluid, we only consider stagnant fluids in our computations. Our numerical model is set up as follows. First, we do not consider bubble nucleation or diffusive growth of the gas bubbles. This simplification is motivated by the fact that fluid dynamical interactions between bubbles become most relevant once the bubbles have reached sufficiently large radii to deform (Bo > 1) [see also Manga and Stone, 1993, 1994, 1995]. At this stage, diffusive bubble growth (limited by the initial volatile content and the bubble number density) is thought to have slowed substantially and starts to come to a halt: For an initial water content of $0.5 \mathrm{wt} \%$, Sparks [1978] estimated a maximum bubble radius of $\sim 5 \mathrm{~mm}$. Similarly, Proussevitch and Sahagian [1996] obtained a maximum size of $6-8 \mathrm{~mm}$ for bubbles originating from magma with $1.52-3.03$ wt \% water. Decompression growth is incorporated in our model (see equation (8)).

[23] Second, all of our simulations are isothermal, because our computational domain is only several bubble radii in size. On such small spatial scales, temperature is typically not expected to vary dramatically. In addition, we note (1) that Sparks [1978] demonstrated that the expansion of gas bubbles by decompression can be approximated as an isothermal process due to a balance of cooling resulting from work done in expanding and conductive heat transfer and (2) that a vertical temperature gradient is unlikely to have an important effect on bubble stability [Rother and Davis, 2005].

[24] Third, we assume flow to be incompressible in both the liquid magma and the gas. Although gas compressibility plays a significant role for the dynamics on the length scale of the entire conduit, its effect at the scale of the bubble radius is small. Very large gas pockets are possible exceptions, and our simulations might underestimate their expansion. While further study is needed to anticipate the dynamic consequences in detail, two recent numerical studies [Crowdy, 2003; Pozrikidis, 2001] indicate that taking the gas compressibility into account will tend to destabilize gas bubbles. Thus, our estimates of maximum bubble size and breakup time are likely upper bound estimates.

[25] Fourth, we assume that both the volatile phase and the surrounding basaltic magma have Newtonian rheology (section 2). For the gas phase, this assumption is straightforward. The Newtonian behavior of basaltic melts has been well established from experiments [e.g., Shaw, 1969; Ryerson et al., 1988]. Rheological complexities arising from the presence of gas bubbles in the melt are fully resolved in our simulations. The effect of crystals, however, is only included by defining an effective viscosity, which implies that the crystals are assumed to be small compared to the bubbles.

[26] Finally, by basing our simulations on the Navier-Stokes equation, we model both phases as continua. Molecular interactions such as the effect of surfactants (i.e., "surface active agents") are not resolved. Although they may play an important role just prior to coalescence or breakup, their properties are essentially unknown for gas bubbles in basaltic magma [Sparks, 1978]. In the absence of resolving the details of the molecular interactions upon microscopic approach of two interfaces, we "define" coalescence to occur in our simulations once the distance between two interfaces has decreased to below grid resolution $\Delta x$. This coalescence condition is corroborated by field studies that have shown that thin magmatic films become unstable in the $\mu \mathrm{m}$ range [Klug et al., 2002].

[27] Before proceeding to the specific setup of our simulations, a few comments on comparing simulations with different dimensions might be in order. We argue that there is a merit in performing both two- and three-dimensional computations. While simulations in three dimensions are appealing for being a more realistic representation of the 
physical problem at hand, two-dimensional simulations offer the possibility of resolving the dynamics with greater accuracy because they can be performed at higher numerical resolution. However, caution is advised in comparing interface dynamics in two and three dimensions, not only because of differences in the flow field (the direct three-dimensional analogue of a two-dimensional bubble is a cylinder of infinite extent, not a sphere), but also because surface energy scales as $\sim \mathrm{ML} / \mathrm{T}^{2}$ in two dimensions and as $\sim \mathrm{ML}^{2} / \mathrm{T}^{2}$ in three dimensions. Kinetic energy on the other hand, scales as $\sim \mathrm{ML}^{2} / \mathrm{T}^{2}$ in both cases. Thus, the scaling of a fluid dynamical system might differ notably between two and three dimensions.

\subsection{Stability Analysis of Isolated Gas Bubbles}

[28] We investigate the shape and stability of an isolated gas bubble rising in basaltic magma over the three nondimensional regimes identified in Figure 1. The boundary conditions are "no-slip" on top and bottom and "periodic" on the sides, implying that an infinite number of bubbles are juxtaposed in the conduit. The viscosity ratio is set to $\Pi_{1}=10^{-6}$ in all simulations.

[29] The setup of the computation implicitly assumes that the gas flux in the conduit is low enough for isolated gas bubbles to exist in the flow. The maximum gas flux to which this scenario applies is approximately given by the gas flux in the modeled computational domain. Out of the three types of simulations considered in this paper, the case of isolated gas bubbles implies the lowest overall gas flux.

\subsection{Impact of Multibubble Interactions on Stability and Interface Dynamics}

[30] The second sequence of simulations aims at constraining the role of the dynamic interaction between multiple bubbles on coalescence, breakup, and deformation over the three nondimensional regimes in Figure 1. In addition to the nondimensional numbers derived in section 2 , we use the bubble size ratio $\Pi_{2}=a_{1} / a_{2}$ and the nondimensional separation distances in vertical and horizontal direction, $\Pi_{3}=$ $d_{z} / a_{1}$ and $\Pi_{4}=d_{x} / a_{1}$, to characterize the different initial conditions. We focus on interactions between bubbles that are comparable in size, i.e., $\Pi_{2}=O(1)$. If the radius of one of the bubbles is significantly larger than the other, the large difference in rise speed will limit the timescale of interaction. Similarly, we consider sufficiently small separation distances, i.e., $\Pi_{3}=O(1)$ and $\Pi_{4}=O(1)$. Analogous to the case of an isolated bubble, we use "periodic" boundary conditions on both sides and "no slip" on top and bottom. Due to the presence of multiple bubbles in the computational domain, the gas flux in the model system is several times higher than that assumed in the previous case (section 4.2).

\subsection{Stability Analysis of Conduit-Filling Gas Pockets}

[31] If the gas flux in the magmatic system is increased further as compared to the low (section 4.2) and moderate (section 4.3) gas fluxes considered so far, bubbly flow will transform into slug flow [e.g., Mudde, 2005; Lucas et al., 2005]. In the slug regime, flow is no longer dominated by bubbles, but instead by conduit-filling pockets of gas. For clarification purposes, we note that in the engineering literature these conduit-filling pockets of gas are referred to as "Taylor bubbles" while "slug" denotes the bubbly mixture in between. In contrast, it is common in the geophysical literature to use "slug" to refer to the conduit-filling gas pocket itself. We abide by the convention of the geophysical literature.

[32] The third group of simulations is targeted at investigating the stability and dynamic behavior of slugs. A key difference of this case compared to the computations outlined in sections 4.2 and 4.3 is that while the first two sequences of simulations are independent of the conduit geometry, we now have to assume an explicit conduit geometry: We model the conduit as being vertical with rectangular "no-slip" walls.

[33] In order to track the behavior of the gas slug over a longer segment of the conduit, we define more elongated computational domains. The nondimensional number $\Pi_{5}=$ $d_{s} / w=O(1)$ captures the relative size of the initial diameter of the gas pocket, $d_{s}$, to the conduit width, $w$. We continue to use a spherical initial condition because it is the most stable.

\section{Results}

\subsection{Dynamic Instability of Isolated Gas Bubbles During Ascent}

[34] A spherical drop is an exact steady solution to the Stokes equation [Batchelor, 2000]. Kojima et al. [1984] were the first to investigate the stability of this solution experimentally and demonstrated that small disturbances to the spherical shape of a dynamical drop can lead to highly distorted shapes, even in the Stokes regime. These findings were confirmed soon afterward through numerical studies [Koh and Leal, 1989; Pozrikidis, 1990]. In agreement with these investigations, we observe that gas bubbles stabilize to a spherical shape in the first regime $(B o<1$ and $R e<1)$ independent of the initial perturbations to the interface. In the second regime $(B o>1$ and $\operatorname{Re}<1)$, however, a wide range of distorted interfaces can develop, depending on the initial condition of the bubble. In the extreme case, the bubble can break up if either the initial distortions of the interface are sufficiently large or if surface tension is negligible. These findings are in agreement with prior studies [Pozrikidis, 1990].

[35] Under flow conditions representative of the third regime ( $B o>1$ and $R e>1$ ), the breakup of an isolated bubble occurs even in the absence of any distortions of the initial interface position. We performed a total of 95 simulations in this regime (Figure 3). We observe two breakup mechanisms of an initially spherical bubble during buoyancydriven ascent: (1) gradual breakup in which small droplets are torn off the rear of the bubble (Figure $4 \mathrm{~b}$ ) and (2) catastrophic breakup in which the bubble collapses rapidly into a large number of bubbles with intermediate to small sizes (Figure 4c).

[36] Figure 4a shows a bubble reaching its steady state shape, a dimpled ellipsoidal cap [e.g., Clift et al., 2005], without breaking up. Figure $4 \mathrm{~b}$ illustrates a gradual breakup sequence. As the bubble deforms into steady state, the interface thins out predominantly at the periphery of its trailing surface. A thin layer of gas develops which eventually becomes unstable and is torn up into several small droplets. The sizes and number of these satellite drops increase with $R e$. Both the formation of a thin sheet of gas trailing the bubble (referred to as "skirt") and the breaking off of small satellite drops are commonly observed in experiments [e.g., Wegener and Parlange, 1973, and references therein]. An 


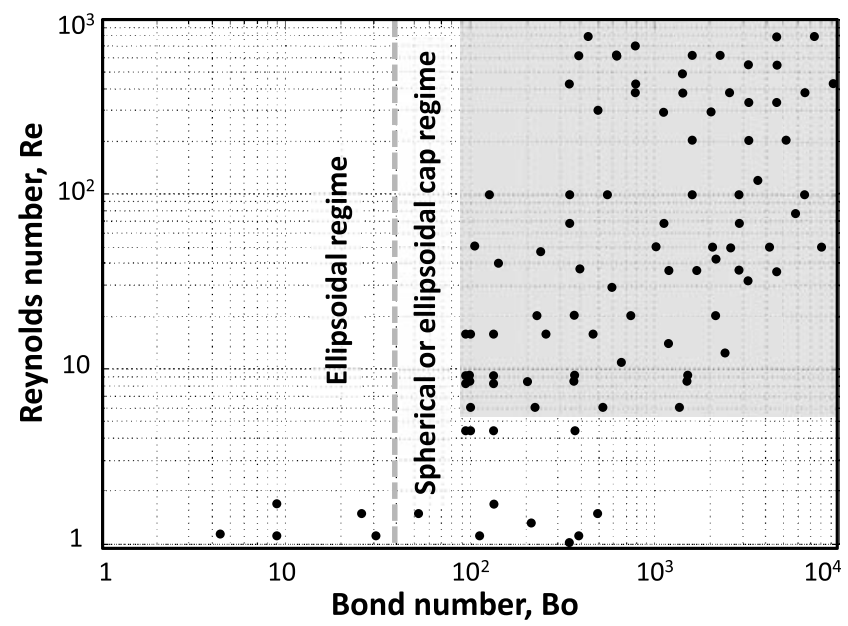

Figure 3. Overview of the nondimensional regime covered by the 95 simulations (black dots) constraining the stability of isolated gas bubbles at $R e>1$ (section 4.2). The gray shading delimits the domain in which we observe breakup in two dimensions. A comparison with the shape regimes for bubbles during buoyant ascent [Grace et al., 1976] confirms that we reproduce the expected steady state shape correctly in our computations. Note that we did not perform simulations for low $B o$ and high $R e$ because that case is of little relevance for basaltic magmas.

extreme example of catastrophic breakup in two dimensions is illustrated in Figure 4c. This mechanism initially resembles the gradual variant: A small dimple forms at the rear of the bubble and grows rapidly in magnitude over time. Contrary to the case of gradual breakup, however, the leading surface of the bubble also begins to deform soon after the onset of motion and small-wavelength perturbations $\lambda \ll a$ are apparent well before breakup (Figure 4c, third panel). Eventually, the gas bubble breaks up, first along the perturbations on the leading surface (Figure $4 \mathrm{c}$, fourth panel) and subsequently along the sides (Figure $4 \mathrm{c}$, fifth to eighth panels).

[37] In three dimensions, we observe the same sequence of breakup regimes from gradual to catastrophic. In the case of gradual breakup, a ring of smaller droplets is torn off the rear of the original bubble as it deforms into its steady state shape. During catastrophic breakup, the rapid growth of the dimple in the rear of the bubble first leads to the formation of a torus which then breaks apart azimuthally into several bubbles (Figure 5). This type of breakup (i.e., torus formation and subsequent azimuthal breakup) has been observed both numerically and experimentally for sedimenting drops at low to intermediate Re [e.g., Machu et al., 2001]. Interestingly, we find that for a given $B o$, the onset of catastrophic breakup occurs at much lower $R e$ in three dimensions compared to two dimensions.

[38] An analysis of our simulations indicates that bubble breakup in basaltic systems is controlled primarily by $R e$. The other nondimensional numbers play a minor role, although they may delay or enhance breakup slightly. The onset of gradual breakup occurs approximately at $\operatorname{Re}>5$. With increasing $\operatorname{Re}(\operatorname{Re} \approx O(10))$, the location of initial breakup shifts from the sides of the bubble surface more and more
A. No breakup

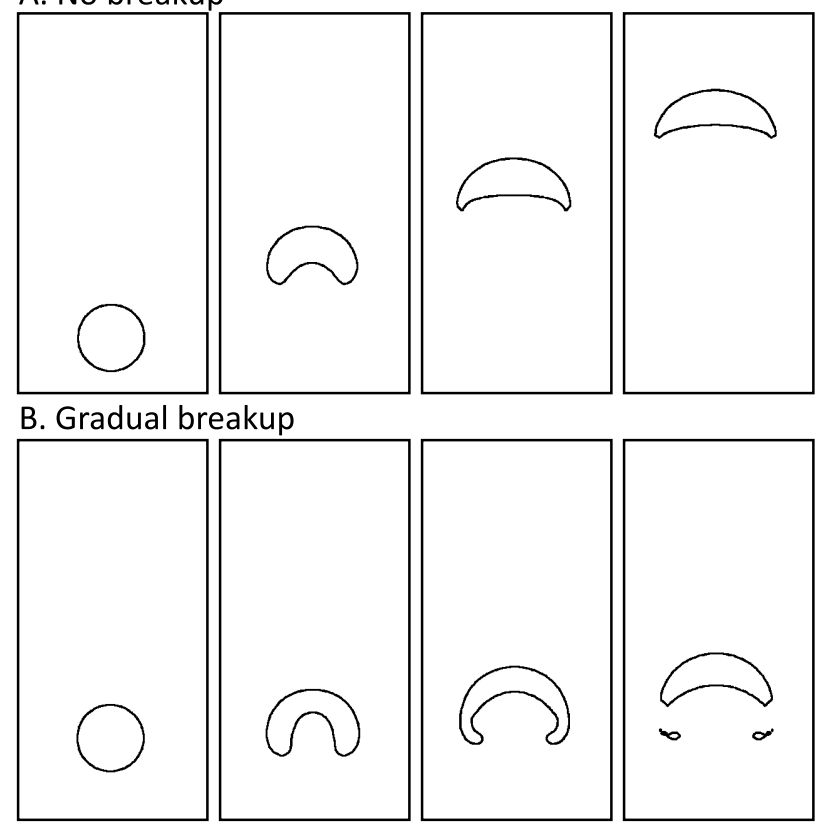

C. Catastrophic breakup

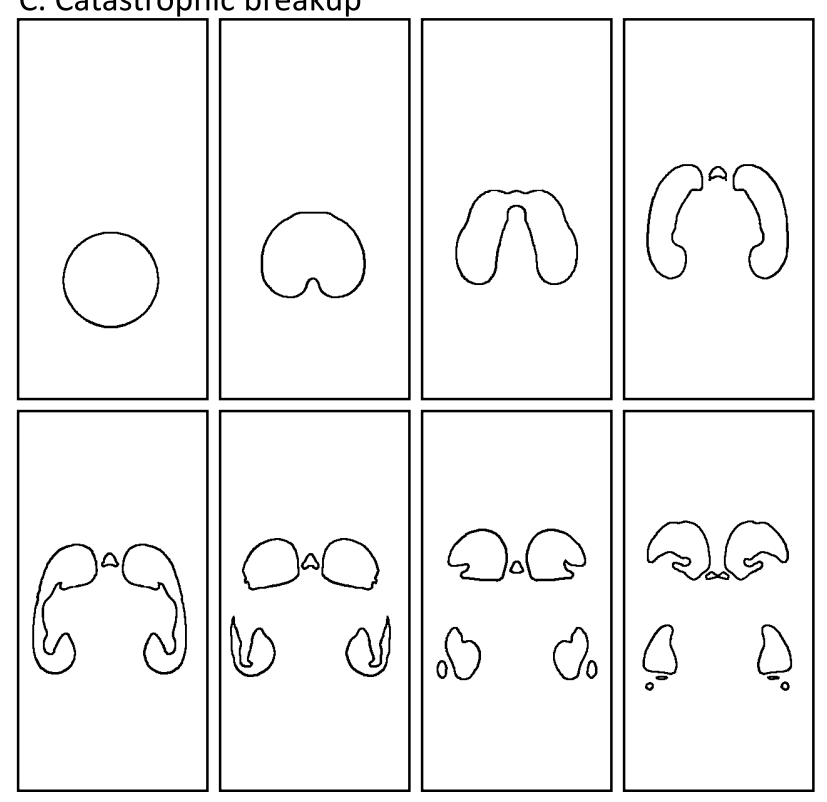

Figure 4. Breakup modes of an initially spherical bubble in two dimensions. (a) An initially spherical bubble reaches its steady state shape without breakup $(R e \approx 5, F r \approx 0.4$, $W e \approx 90$, and $\Pi_{1}=10^{-6}$ ). The snapshots shown are at nondimensional times $t=0,1.97,3.93$, and 5.90. (b) Gradual breakup during which three small bubbles are torn off each side. The run is characterized by $\operatorname{Re} \approx 25, \mathrm{Fr} \approx 0.3, \mathrm{We} \approx$ 800 , and $\Pi_{1}=10^{-6}$. Snapshots are at $t=0,1.42,2.08$, and 2.48. (c) An extreme example of breakup in the catastrophic regime characterized by $R e \approx 250, F r \approx 0.16$, $W e \approx 1350$, and $\Pi_{1}=10^{-6}$. Snapshots are shown for nondimensional times $t=0,0.59,1.03,1.58,1.91,2.04,2.26$, and 2.49. The exact sequence of catastrophic breakup is highly dependent on both material parameters and grid resolution, particularly after the initial rupturing of the upper surface of the bubble. The grid resolution in all runs is $80 \times 160$. 

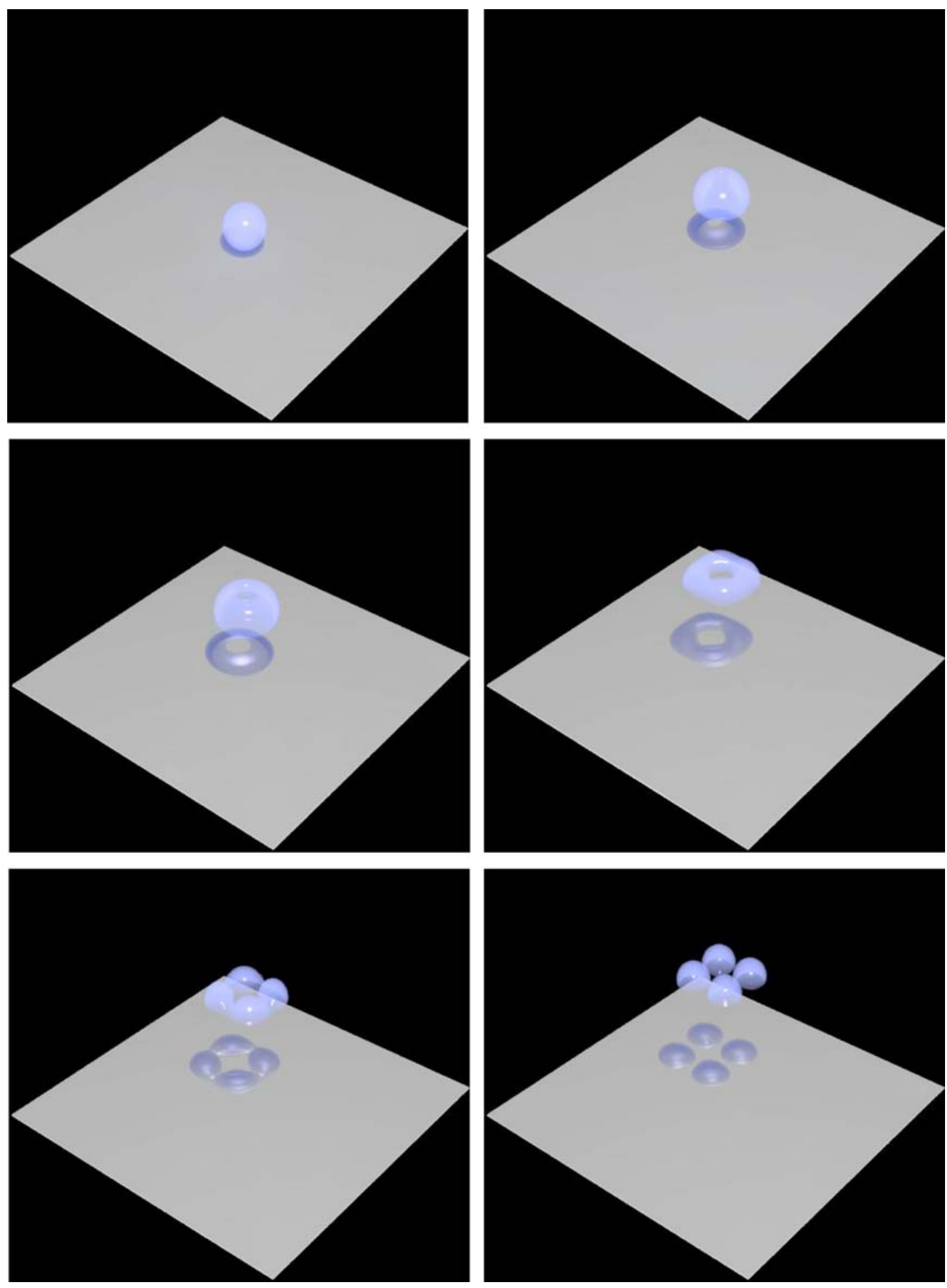

Figure 5. Catastrophic breakup of an initially spherical bubble in three dimensions. The nondimensional numbers are $R e \approx 16, B o \approx 1150$, and $\Pi_{1}=10^{-6}$. We stress that analogous to the two-dimensional case (Figure 4c), it is likely that a series of small bubbles is generated during catastrophic breakup in three dimensions, but is not resolved in three dimensions because of resolution restrictions. The computation was performed in a three-dimensional domain with a grid resolution of $75 \times 75 \times 50$ equidimensional cells. Thus, the resolution in this three-dimensional computation is comparable to the two-dimensional simulation in Figure 4c. The snapshots shown refer to nondimensional times $t=0,1.15,1.73,2.88,3.45$, and 4.3. Note that the gray square on the bottom is displayed for visualization purposes only. It does not indicate the size of the computational domain. 


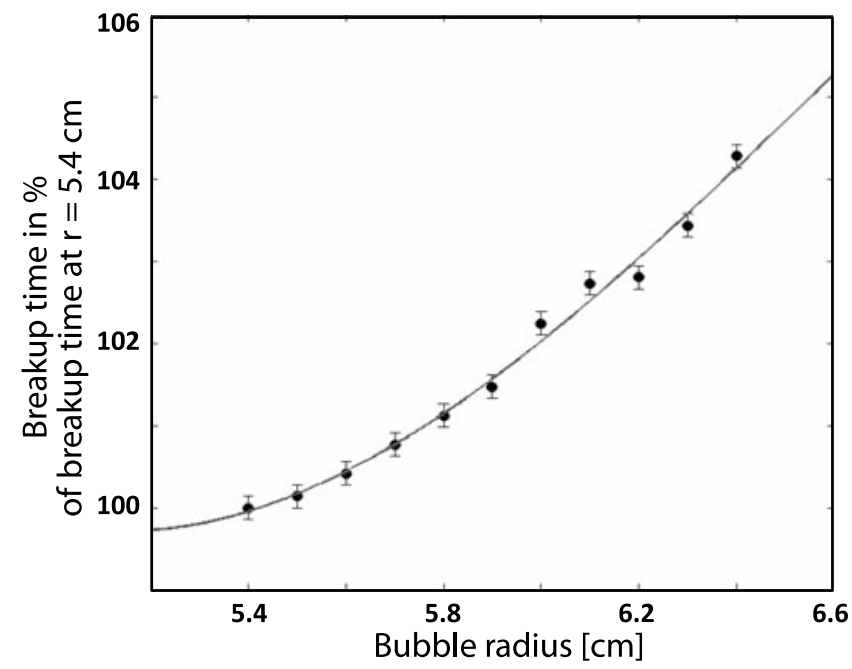

Figure 6. Dependence of the breakup time $t_{B}$ on bubble radius $a$ for gradual breakup. In order to evaluate the dependence of $t_{B}$, the computation is dimensional with $\rho_{f}=$ $3500 \mathrm{~kg} / \mathrm{m}^{3}, \rho_{g}=1.226 \mathrm{~kg} / \mathrm{m}^{3}, g=9.81 \mathrm{~m} / \mathrm{s}^{2}, \sigma=0.3 \mathrm{~N} / \mathrm{m}$, $\mu_{f}=10 \mathrm{~Pa} \mathrm{~s}$, and $\mu_{g}=10^{-5} \mathrm{~Pa} \mathrm{~s}$. The breakup time is expressed in percent of the initial breakup time at $a=$ $5.4 \mathrm{~cm}$. The numerical data points are associated with an error bar reflecting the finite time step $\Delta t$ in the computation. The line represents a cubic fit through the data.

upward and the sizes and number of broken off bubbles increase. Thus, breakup becomes increasingly "efficient" at intermediate $R e$, by which we mean that the radius of the largest bubble after breakup is significantly smaller than the original bubble radius. If $R e$ exceeds the onset of breakup $(R e \approx 5)$ by more than an order of magnitude, the mode of breakup changes to catastrophic. During a catastrophic breakup sequence, the original radius of the bubble is reduced dramatically. For example, the area of the largest bubble after breakup in Figure $4 \mathrm{c}$ is only $~ 33 \%$ of the initial bubble area.

[39] In both two and three dimensions, we find that for a given set of material parameters of magma and gas, the tendency for a bubble to break up depends sensitively on its size. This strong size dependence of the breakup time can be understood through the following simple scaling argument. The relevant physical parameters for breakup are bubble radius $a \sim \mathrm{L}$, surface tension $\sigma \sim \mathrm{M} / \mathrm{T}^{2}$, density difference $\Delta \rho \sim \mathrm{M} / \mathrm{L}^{3}$, dynamic viscosity $\mu \sim \mathrm{M} / \mathrm{LT}$, and gravitational acceleration $g \sim \mathrm{L} / \mathrm{T}^{2}$ (where $\mathrm{M}$ stands for mass, $\mathrm{L}$ for length, and $\mathrm{T}$ for time) yielding

$$
t_{B} \sim \frac{\sigma a^{3} \Delta \rho^{2}}{\mu^{3}},
$$

when requiring that $t_{B} \sim \mathrm{T}$. We are able to confirm the hypothesized dependence of breakup time on bubble radius $t_{B} \sim a^{3}$ only for gradual breakup and only during its initial stages ( $R e \ll O(100)$, see Figure 6). At larger $R e$, we observe a more complex correlation between breakup time and bubble radius as detailed in the auxiliary material. Independent of the exact scaling of $t_{B}$ with $a$, however, breakup occurs very rapidly, typically before the bubble has traveled a distance comparable to its own diameter.

\subsection{Effect of Multibubble Interactions on Breakup}

[40] At higher gas fluxes than those considered in section 5.1, the interaction between bubbles plays an increasingly important role. This is particularly true when bubbles are deformable, i.e., in regimes two $(B o>1$ and $R e<1)$ and three $(B o>1$ and $R e>1)$. One well-known consequence of bubble deformability is enhanced coalescence in the Stokes regime [Manga and Stone, 1993, 1994, 1995]. In this section we demonstrate that coalescence is only one aspect of multibubble interactions because deformability enhances not only coalescence but also breakup.

[41] Since multibubble interactions play a marginal role for interface dynamics in the first regime $(B o<1$ and $R e<1)$, we will not discuss this regime further. The second regime ( $B o>1$ and $R e<1)$ is more interesting, because, while small deformations impede coalescence [Rother et al., 1997; Rother and Davis, 2001], large deformations enhance coalescence [Manga and Stone, 1993, 1994, 1995]. For easily deformable bubbles $(B o>50)$ of comparable size $\left(\Pi_{2}=O(1)\right)$, multibubble interactions can manifest themselves in three ways in the Stokes regime, depending on $B o$ and the nondimensional separation distance $\Pi_{4}$ as analyzed in detail by Zinchenko et al. [1999]: First, at large horizontal distances $\Pi_{4}$, the two bubbles will pass each other without topological change of the interface. Second, in a certain range of horizontal distances $\Pi_{4}$, the interaction of the bubbles will lead to breakup of either one or both bubbles. Typically, breakup proceeds by a small drop being torn off an ascending bubble because of the presence of a trailing drop [Zinchenko et al., 1997, 1999; Davis, 1999; Kushner et al., 2001]. Third, at even smaller distances $\Pi_{4}$, the two bubbles coalesce with each other [Manga and Stone, 1993, 1994, 1995].

[42] In our investigation of multibubble interactions at finite $R e$, we focus on bubbles in the stable regime $(R e<$ $O(10))$. The reason is that bubbles in the unstable size range will be rare in a typical flow field, because of their rapid breakup (section 5.1). Figure 7 gives an overview of the nondimensional range covered by our simulations. Qualitatively, we observe a similar range of dynamic interactions at low $R e$ as compared to $R e=0$ [Zinchenko et al., 1999]. Quantitatively, however, we find that the range of nondimensional separation distances over which coalescence occurs decreases with increasing $R e$. For example, at intermediate $(R e \approx O(10))$, two bubbles coalesce only if they lie approximately within the line of ascent of each other (i.e., $\left.\Pi_{4}<1.2\right)$. At low $\operatorname{Re}(\operatorname{Re} \approx O(1))$, coalescence is observed over a much wider range of initial separation distances. Figure 8 shows an example of a three-dimensional computation of two deformable bubbles passing each other despite their small initial separation. It should be noted at this point that three-dimensional computation of bubble interactions are challenging because of the high resolution required to accurately resolve the fluid dynamics in the thin magmatic film separating the two bubbles. Therefore, we primarily focus on two dimensions in this section. Please refer to the auxiliary material for a more detailed discussion or resolution restrictions.

[43] On the basis of our simulations, we identify two other important characteristics of multibubble interactions 


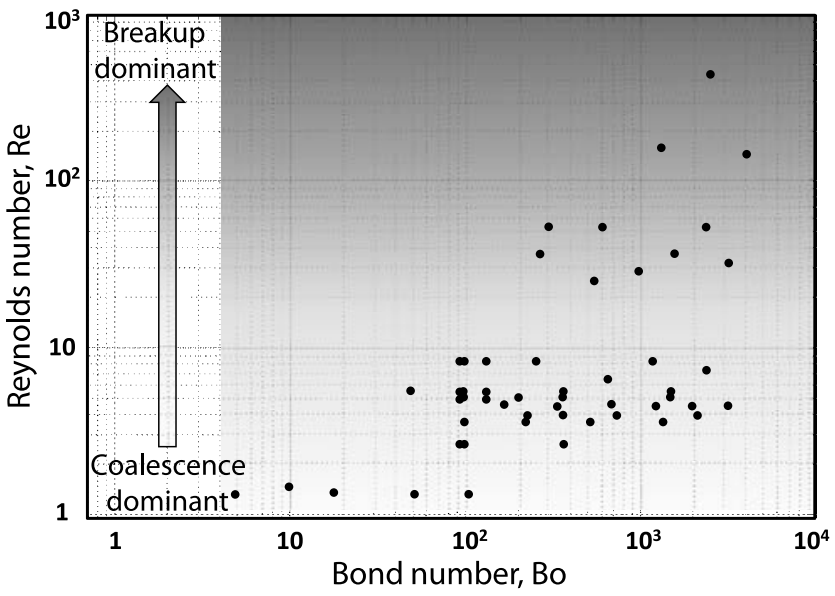

Figure 7. Overview of the nondimensional regime covered by 52 simulations (black dots) investigating the effect of multibubble interactions on breakup at $R e>0$ (section 4.3). The computations have been binned based on their initial conditions, where $1.1 \leq \Pi_{2} \leq 2.0,1.1 \leq \Pi_{3} \leq 2.0$ and $1.1 \leq$ $\Pi_{4} \leq 3.0$. The gray shading visualizes our finding that while coalescence and breakup commonly occur in sequence at $R e>0$, coalescence dominates at small $R e$ and breakup at large $R e$. at finite $R e$. First, we are no longer able to identify a "typical" breakup sequence as for the case of an isolated bubble (section 5.1) or in the Stokes regime [Zinchenko et al., 1999]. Figure 9 shows two examples of breakup at $R e>0$ for slightly different horizontal separation distances $\left(\Pi_{4}=2\right.$ (Figure 9a) and $\Pi_{4}=1.8$ (Figure 9b)), highlighting that the exact breakup process is very sensitive to initial conditions. Second, coalescence and breakup are clearly not mutually exclusive. In fact, we find that breakup and coalescence occur in sequence in the majority of simulations, sometimes repeatedly (see Figure 9b). The balance between coalescence and breakup depends primarily on $\operatorname{Re}$ (as long as $B o \approx O(100)$ or larger) with coalescence more commonly observed at small $R e$ and breakup more commonly observed at large $R e$ (see Figure 7).

[44] One important ramification of the observed balance between coalescence and breakup is that multibubble interactions can destabilize bubbles that would not have broken up when isolated, even in the Stokes regime as long as $B o$ is sufficiently high $(B o>10)$. A comparison of the timescale of breakup induced by multibubble interactions to that of breakup for isolated bubbles indicates that unstable bubbles break up more rapidly than they interact (see Figure 9).
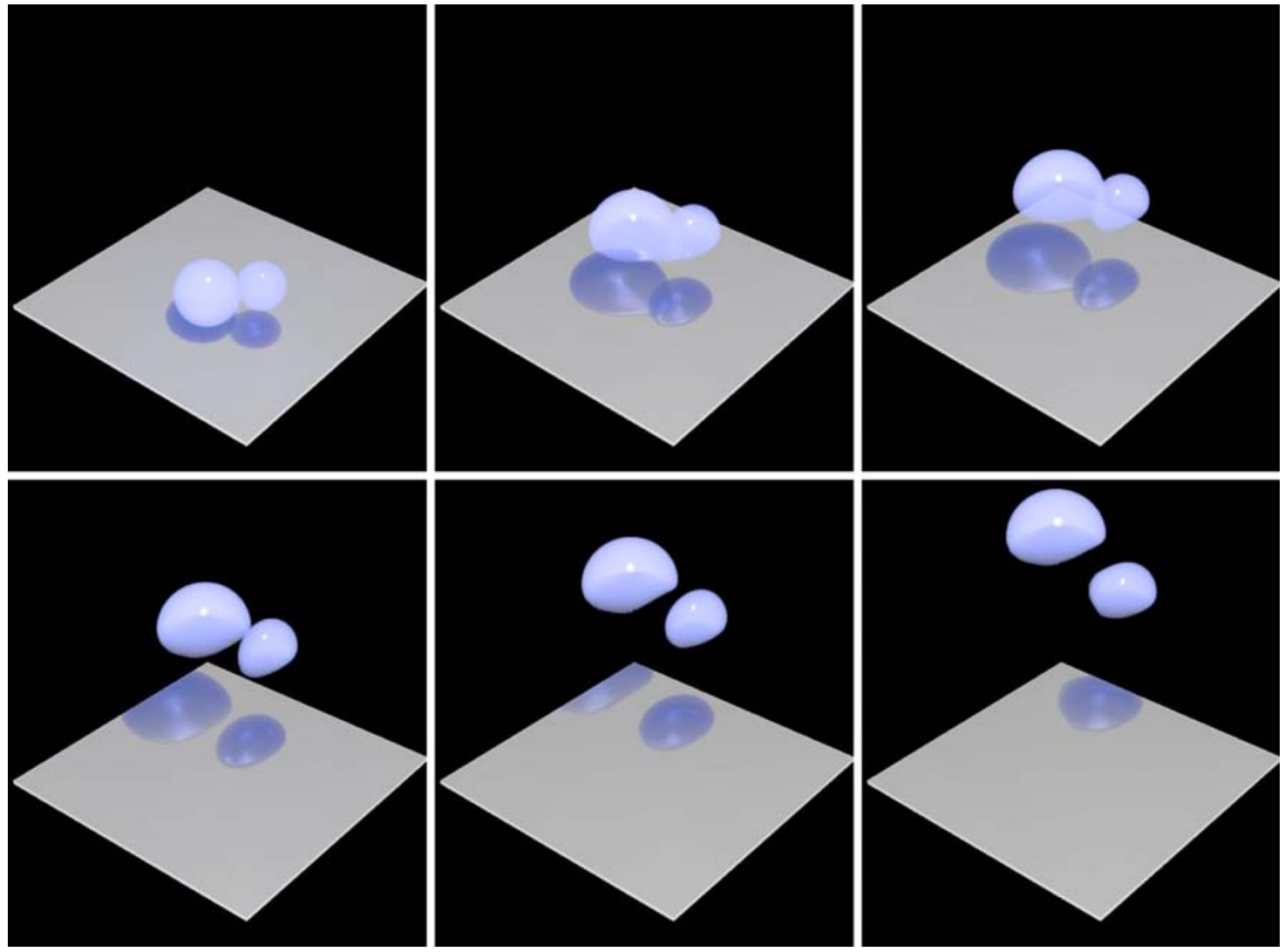

Figure 8. A three-dimensional computation of two deformable bubbles coming into close contact without coalescing. Nondimensional numbers are $R e \approx 2, B o \approx 166, \Pi_{1}=10^{-6}, \Pi_{2}=1.43, \Pi_{3}=1.2$, and $\Pi_{4}=2.0$. Snapshots are at $t=0,2.04,3.06,4.08,5.10$, and 6.12. The resolution is $50 \times 50 \times 80$. 
A. Sequence of breakup

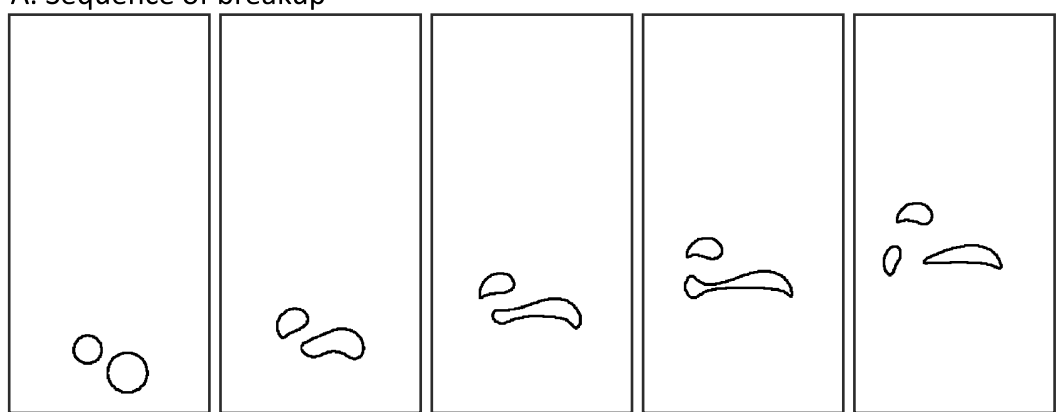

B. Sequence of coalescence and breakup

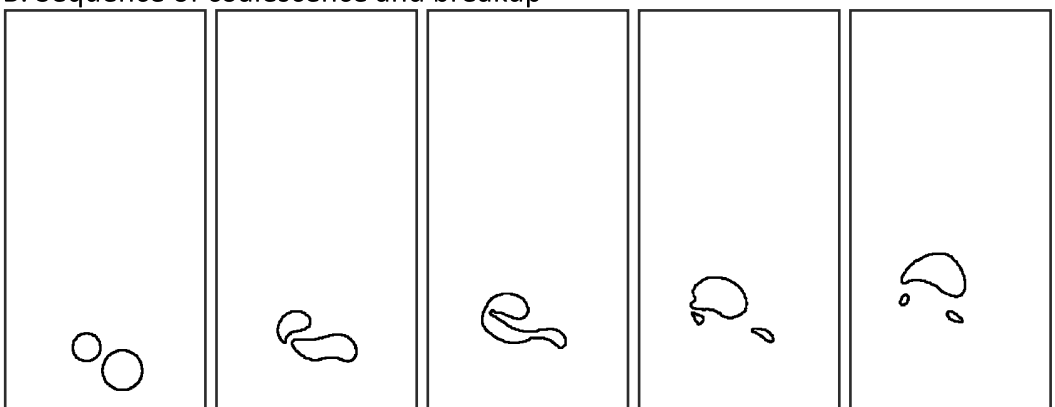

C. Sequence of breakup for unstable bubbles

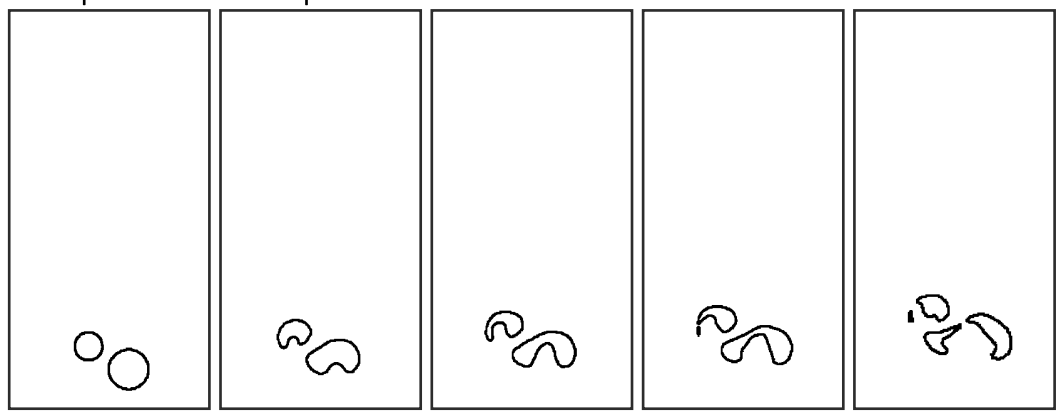

Figure 9. Two breakup sequences as a consequence of differing initial separation distances in the vertical direction (a) $\Pi_{4}=2$ and (b) $\Pi_{4}=1.8$. Both computations are based on $\operatorname{Re} \approx 5, \mathrm{Fr} \approx 0.2, \mathrm{We} \approx 70, \Pi_{1}=10^{-6}$, $\Pi_{2}=1.43$, and $\Pi_{3}=1.16$. The snapshots shown for the first computation (Figure 9a) refer to nondimensional times $t=0,1.5,3.0,4.5$, and 6.0. Those in Figure $9 \mathrm{~b}$ refer to $t=0,2.32,3.48,4.41$, and 5.80. (c) Multibubble interactions for bubbles in the unstable size range (section 5.1). Nondimensional numbers are $\operatorname{Re} \approx 350$, $F r \approx 0.2, W e \approx 3000, \Pi_{1}=10^{-6}, \Pi_{2}=1.43, \Pi_{3}=1.16$, and $\Pi_{4}=2$. Snapshots are at nondimensional times $t=0,0.34,0.51,0.64$, and 0.87 . Initially, the deformation of each bubble is reminiscent of the breakup sequence of isolated bubbles (Figure 4). The presence of the other bubble only becomes apparent during the late stages of breakup, indicating that unstable bubbles break up more rapidly than they interact. All three computations are based on a grid resolution of $100 \times 200$.

\subsection{Dynamic Instability of Slugs}

[45] As mentioned previously (section 4.4), slugs scale differently than isolated bubbles, implying that the three scaling regimes illustrated in Figure 1 are no longer necessarily meaningful. Most importantly, the limiting case of high surface tension forces (Figure 1, regime 1) does not apply, because slugs only move if they are sufficiently deformable, namely, if

$$
\frac{\rho_{f} g a^{2}}{\sigma} \approx B o>0.842
$$

as has been determined experimentally [Bretherton, 1961]. Thus, a diameter of several millimeters is typically required for slugs in basaltic magma to ascend under their own buoyancy.

[46] Since our main goal is to investigate the stability of conduit-filling slugs, we focus on $R e>1$ (Figure 10). Analogous to the case of isolated gas bubbles (section 5.1), we observe two regimes of breakup, gradual and catastrophic (Figure 11). Gradual breakup results in small droplets being torn off the gas pocket in the vicinity of the walls. Contrary to the case of isolated gas bubbles, where multiple droplets are commonly torn off the original bubble simultaneously (section 5.1), gradual breakup of slugs tends to occur repeatedly. Figure 11a shows an example of a single sequence of gradual breakup, and Figure $11 \mathrm{~b}$ shows a double sequence of gradual breakup. We observe repeated breakup for most 


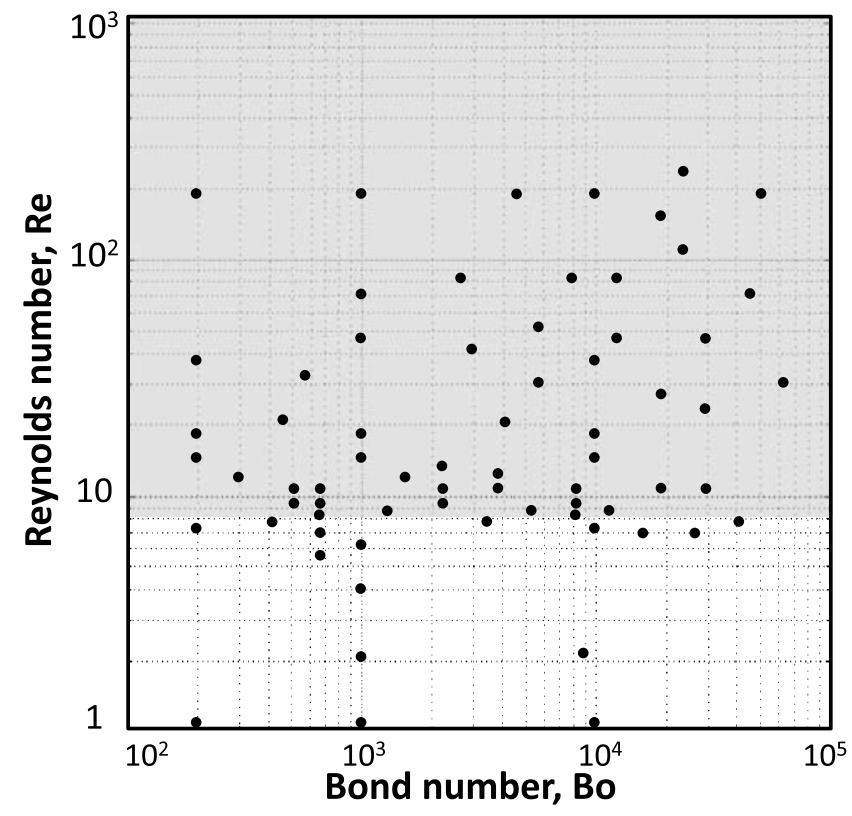

Figure 10. Overview of the nondimensional regime covered by 69 simulations (black dots) to constrain the stability of gas slugs (section 4.4). For all computations, $\Pi_{1}=10^{-6}$ and $\Pi_{5}=0.7$. The gray shading delimits the domain in which we observe breakup in two dimensions.

slugs in the unstable regime. The number of breakup cycles and the size of torn off bubbles increase with $R e$.

[47] An extreme case of catastrophic breakup is illustrated in Figure 11c. Initially, it proceeds similarly to the case of an isolated bubble: A dimple forms in the rear of the slug and grows over time. However, the dimple does not reach the upper surface of the slug because of the large volume of gas confined in a narrow conduit. Instead, rupture akin to gradual breakup occurs along the sides. Simultaneously, a Rayleigh-Taylor instability forms on the upper surface, propagates through the gas volume, and leads to breakup of the remaining gas slug into two equally sized segments. Similar to the isolated case, the onset of catastrophic breakup is not discontinuous. Small-wavelength perturbations are apparent on the leading surface of the slug well before the regime of catastrophic breakup is reached. Therefore, cyclical breakup may be interpreted as a more moderate variant of catastrophic breakup.

[48] A special consideration when investigating slug breakup is the vicinity of the solid wall and the role it plays for breakup. Not surprisingly, we find that at $\Pi_{5}>0.8$ breakup is observed at all Re and clearly results from the close proximity of the initial interface to the boundary. However, lowering the fill factor to $\Pi_{5} \leq 0.7$ eliminates this bias. Figure 12 demonstrates that the breakup behavior described in this section is not a boundary artifact. It shows the steady state shapes of three gas slugs of equal volume and initial shape at varying $R e$. As expected, the steady state width of the slug increases with increasing $R e$, which leads to an increase in shear stresses in the magmatic film separating the slug from the conduit walls and facilitates dynamic instabilities. To further clarify the role of the proximity of the conduit walls for breakup, we compare the onset of breakup for a gas slug to an isolated gas bubble of identical size. We find that conduit walls have a slightly stabilizing effect, which seems to be primarily the consequence of the lower rise speed of the slug (and potentially also of the different steady state shape of the slug, see Figure 12). Therefore, although we observe slug breakup at approximately the same $R e$ as bubble breakup, this $R e$ is representative of a slightly larger volume of gas in the case of a slug.

\section{Discussion}

\subsection{Comparison of Theoretical and Numerical Constraints on Bubble Breakup}

[49] Despite the seemingly obvious differences between gradual and catastrophic (Figure 4) breakup, we argue that they are both manifestations of the breakup mechanism outlined in section 3. In the case of catastrophic breakup, the connection to the theoretical breakup model is evident: Small-wavelength perturbations are apparent on the leading surface of the bubble (Figure 4c, second panel) and growth of these Rayleigh-Taylor instabilities eventually leads to breakup (Figure 4c, third panel). For breakup sequences at intermediate $R e$, surface perturbations can still be distinguished visually, although they no longer grow fast enough to cause breakup along the leading surface. The slower growth can be explained by dispersive viscous damping. As plotted in Figure 2, barely unstable wavelengths grow very slowly at high viscosities $(\mu \geq 100 \mathrm{~Pa} \mathrm{~s})$.

[50] The conclusion that both gradual and catastrophic breakup fit well into the theoretical framework (section 3) warrants a brief comparison of predicted (Table 1) to computed maximum stable bubble sizes. We perform this comparison only in two dimensions because the semiempirical model itself relies on a linearized stability analysis in two dimensions [Grace et al., 1978; Plesset and Whipple, 1974]. In our computations, we use the same physical parameters as in Table 1 with $\mu_{f}=10 \mathrm{~Pa}$ s. We find good agreement between predicted and numerical results. The bubble size, $a_{\text {grad }}$, that marks the onset of the regime of gradual breakup is smaller than the predicted maximum bubble size by approximately $a_{\text {grad }} \approx 0.85 a_{\text {max }}$. Clearly, the critical radius for the onset of gradual breakup is not a very good proxy for the maximum stable bubble size, because the size of the bubble changes only marginally during this moderate variant of breakup. The extreme form of catastrophic breakup is observed for bubble radii of $a_{\text {cat }} \approx 2.2 a_{\text {max }}$. During these more violent breakup sequences, the initial bubble size is typically reduced by at least $50 \%$, leaving a bubble of approximately the size predicted in Table 1.

[51] A noteworthy deviation of our simulations from the breakup model (section 4.1) is that instabilities do not necessarily disappear once they have reached the periphery of the bubble surface, as would be expected for a RayleighTaylor instability. The cyclical breakup of gas slugs in Figure $11 \mathrm{~b}$ is an example. We hypothesize that breakup in this case might be the result of the combined effect of a Rayleigh-Taylor and a Kelvin-Helmholtz instability. As the initial Rayleigh-Taylor instability is advected along the leading surface of the slug, it approaches the conduit walls, and shearing intensifies. The surface perturbation may then grow through a Kelvin-Helmholtz instability and eventually 


\section{A. Gradual breakup}
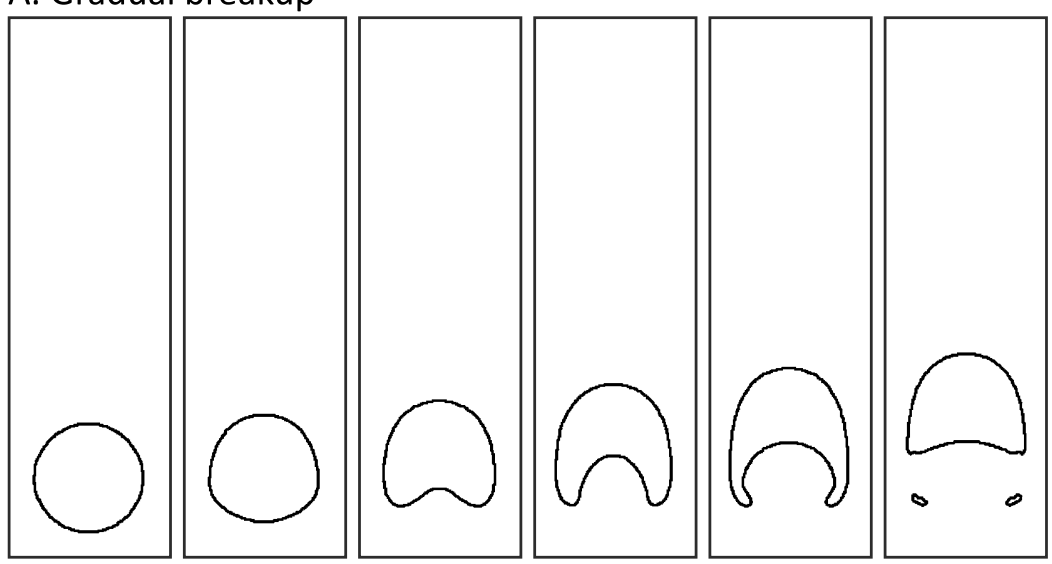

B. Cyclical variant of gradual breakup
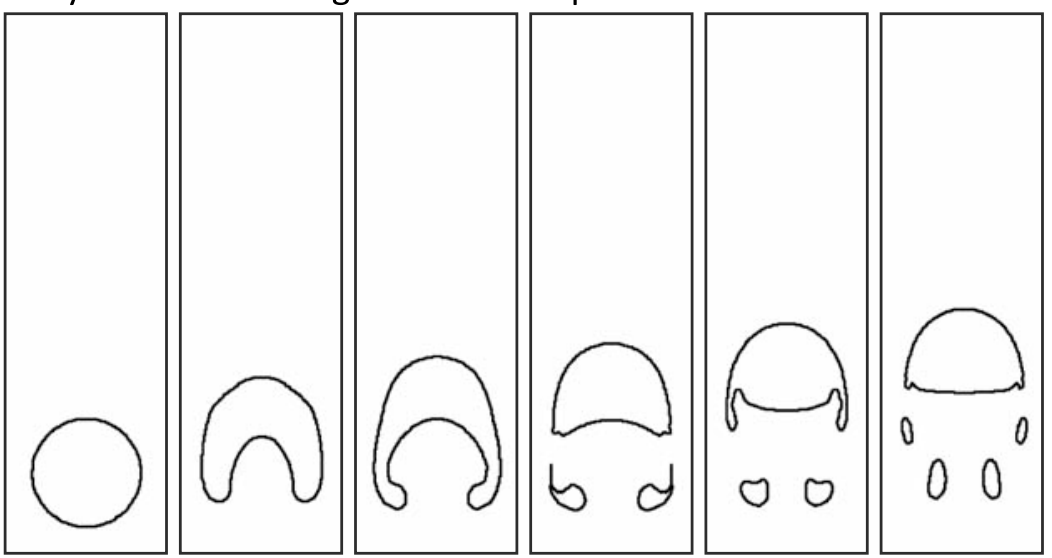

\section{Catastrophic breakup}
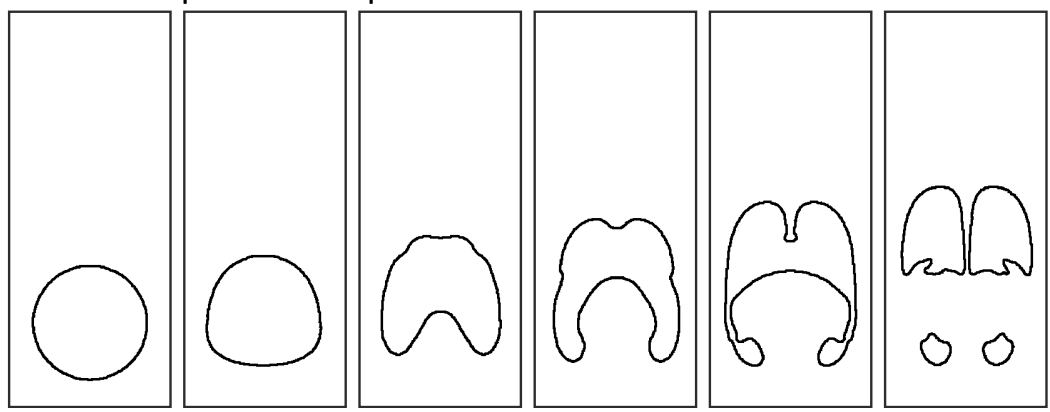

Figure 11. Breakup modes of an initially spherical gas slug in two dimensions. (a) During gradual breakup, small bubbles are torn off the rear of the slug analogous to the case of an isolated bubble (Figure 4b). The nondimensional numbers are $\operatorname{Re} \approx 10, \mathrm{Fr} \approx 0.14, W e \approx 95, \Pi_{1}=10^{-6}$, and $\Pi_{5}=0.67$. The snapshots shown are at nondimensional times $t=0,0.30,0.60,0.90,1.20$, and 1.47. (b) Gradual breakup may occur cyclically. The nondimensional numbers for this computation are $\operatorname{Re} \approx 50, F r \approx 0.16$, $W e \approx 100, \Pi_{1}=10^{-6}$, and $\Pi_{5}=0.67$. Snapshots are at nondimensional times $t=0,0.94,1.33,1.56,1.93$, and 2.20. (c) Simultaneous to catastrophic breakup, gradual breakup occurs along the walls. The nondimensional numbers are $R e \approx 80, F r \approx 0.10, W e \approx 3000, \Pi_{1}=10^{-6}$, and $\Pi_{5}=0.7$. The snapshots shown are at $t=0,0.29,0.59,0.88,1.18$, and 1.47. All three computations were performed at grid resolutions of $80 \times 240$.

trigger breakup along the sides of the slug. The onset of a Kelvin-Helmholtz instability is determined by the Richardson number, $R i=F^{-1}$. Although $R i$ for bubbles and slugs in basaltic systems is typically too high to expect the spontaneous formation of a Kelvin-Helmholtz instability, a preexisting perturbation could still grow through this mechanism.

\subsection{Nondimensional Conditions for a Coalescence Cascade}

[52] The RSD model assumes that the gas slugs representative of normal Strombolian activity form through progressive coalescence of gas bubbles. It relies on two main assumptions about the fluid dynamical interactions between 


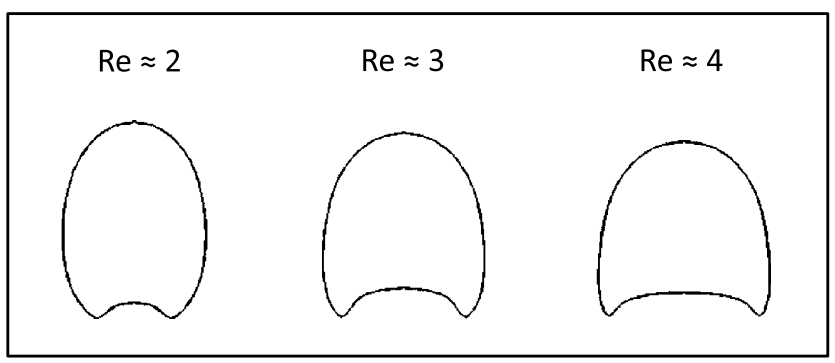

Figure 12. Dependence of the steady state shape of conduit-filling slugs on Re. All computations are based on a spherical initial condition and $\Pi_{5}=0.7$. At finite $R e$, a dimple forms at the rear of the slug visible on all three interfaces. As $R e$ increases from left to right, the steady state width of the slug increases, and the magmatic films separating interfaces and conduit walls are thinned out. Shear stresses will intensify as the magmatic films thin out and eventually lead to the tearing off of small droplets similar to gradual breakup of isolated bubbles (Figure 4b).

magmatic and gaseous phase. (1) It assumes that a large bubble coalesces with all smaller bubbles lying within the vertical line of ascent of the large bubble [Wilson and Head, 1981; Parfitt and Wilson, 1995], and (2) it proposes that coalescences can lead to a runaway situation resulting in the formation of a single large bubble.

[53] On the basis of our numerical experiments, we conclude that the first assumption is justified for deformable bubbles of comparable size $\left(\Pi_{2} \sim O(1)\right)$. In fact, the assumption is rather conservative because the range of initial separation distances in the horizontal direction which result in bubble coalescence is $\Pi_{4}>1$ at $R e<O(10)$ (section 5.2). Judging from our simulations, the second assumption is less straightforward, because both coalescence and breakup are commonly observed in free flow at finite $\operatorname{Re}$ (provided that $B o \gg 1$ ). As discussed in section 5.2, the balance of coalescence and breakup depends primarily on $R e$. Once bubbles grow sufficiently such that $R e>O(10)$, breakup dominates, gradually reducing the average bubble size. In that case, continued breakup and coalescence of bubbles would yield a bubble population with a maximum bubble radius comparable to the critical radius for gradual breakup of isolated bubbles (section 5.1). Evidently, this rationale only applies to free flow, where the average bubble radius $a \ll d$, with $d$ representing the half width of the conduit. We conclude that it is possible for gas slugs to form through a coalescence cascade only if the viscosity is sufficiently high and/or the conduit sufficiently narrow such that bubbles are characterized by $B o>1$ and $\operatorname{Re}<O(1)$ throughout the evolution of the cascade.

[54] For Stromboli, the volume of the slug is thought to be in the range of $20 \mathrm{~m}^{3}$ to $35 \mathrm{~m}^{3}$ [Vergniolle and Brandeis, 1994; Ripepe and Marchetti, 2002]. Since the characteristic radius in our computations is based on the spherical initial condition, a Strombolian slug would thus be represented by an effective radius of several meters $(r \approx 1.7-$ $2.0 \mathrm{~m}$ ). Given the large size of the slug, we estimate that minimum magma viscosities on the order of $\mu_{f} \approx 5 \times 10^{4} \mathrm{~Pa} \mathrm{~s}$ would be required for a coalescence cascade in the upper conduit. Estimates for the viscosity of Strombolian lava vary from 50-500 Pa s [Vergniolle et al., 1996] for the samples acquired by Capaldi et al. [1978] and Francalanci et al. [1989] to $10^{1.5}$ to $10^{4} \mathrm{~Pa} \mathrm{~s}$ in a more recent study evaluating viscosity at Stromboli as a function of temperature and water content [Misiti et al., 2009]. Most commonly, values in the range of $O(100) \mathrm{Pa}$ s are used [e.g., Blackburn et al., 1976; Vergniolle and Brandeis, 1996; Vergniolle et al., 1996]. Nonetheless, it might not be unrealistic to expect that the effective viscosity in the upper conduit is significantly elevated $\left(\mu_{f} \sim O\left(10^{4}\right) \mathrm{Pa}\right.$ s or larger) because of its high phenocryst content [e.g., Bertagnini et al., 1999; Francalanci et al., 1999; Rosi et al., 2000]. An important caveat is that our numerical approach does not resolve the dynamic effect of a high phenocryst density on multibubble interactions or on coalescence/breakup of individual bubbles, which might very well be substantial.

\subsection{Nondimensional Conditions for Stable Slug Rise}

[55] The CF model is based on a series of laboratory experiments [Jaupart and Vergniolle, 1988, 1989] in which the magma chamber is represented as a cylindrical tank and the volcanic conduit as a narrow pipe located in the center of the roof. Gas is introduced into the tank from below and accumulates below the flat top, where it coalesces. Eventually the gas layer empties into the pipe. Depending on the fluid properties in the tank and on the incoming gas flux, three flow regimes are observed in the conduit: bubbly flow (interpreted as the analogue of passive degassing), spherical bubbles with a characteristic spacing (analogue of normal Strombolian type eruptions), and slug flow (analogue of Hawaiian type eruptions). The main assumptions of the CF model are that (1) coalescence occurs entirely in the magma chamber (or, more generally, underneath a large roof area in the conduit) and (2) gas slugs rise stably through the conduit. Only the second assumption lends itself to evaluation through our simulations.

[56] In section 5.3 we demonstrate that slugs are prone to dynamic instabilities if they are characterized by $\operatorname{Re} \approx O(10)$ or larger (section 5.3). Vergniolle and Brandeis [1996] suggest that the gas slugs causing normal activity at Stromboli are characterized by $R e \approx 80$. Our simulations, however, indicate that a slug at $R e \approx 80$ would break up catastrophically within seconds after formation (see Figure 11c).

[57] The three key factors determining Re are (1) the size of the slug, (2) the width of the conduit, and (3) the magma viscosity. Additional considerations, not included in our simulations, are deviations from the assumed vertical conduit geometry (e.g., a local widening of the conduit) and velocity gradients in an ambient magmatic flow field, both of which are expected to reduce slug stability. At least the latter of these two aspects might be important to consider, because acoustic pressure oscillations during the eruption are thought to be related to vigorous vibrations of the slug interface [Vergniolle and Brandeis, 1994].

[58] In order to stabilize the ascent of a Strombolian slug from the magma chamber to the free surface, we estimate that magma viscosities above $\mu_{f} \approx 5 \times 10^{4} \mathrm{~Pa} \mathrm{~s}$ would be required throughout the entire conduit. This conflicts not only with petrological observations indicating lower vis- 
cosities, at least in the deep conduit [Misiti et al., 2009; Bertagnini et al., 2003], but also with acoustic observations [Vergniolle et al., 1996] and the presumed rise speed of a Strombolian slug [e.g., Harris and Ripepe, 2007]. Although the incompatibility of slug stability with the presumed low viscosities in the deep conduit could be reconciled by slug formation at shallow depth, for example in a dike located in the upper conduit [Menand and Phillips, 2007], the disagreement with acoustic data and a presumed rise speed of $10-70 \mathrm{~m} / \mathrm{s}$ [Harris and Ripepe, 2007] remains.

[59] Since the CF model [Jaupart and Vergniolle, 1988, 1989 ] is based on fluid dynamical experiments, it is valuable to briefly assess the reasons why slug instability is not observed in the experiments. We suggest that simulations and experiments scale differently because of the small width $w$ of the laboratory conduit $w \sim O(\mathrm{~cm})$ as compared to the size of the Strombolian conduit $w \sim O(\mathrm{~m})$. Therefore, the slugs in the experiment fall well into the stable size range (see Table 1 and Figure 2), but Strombolian slugs might not. Also, Jaupart and Vergniolle [1989] investigated variations in surface tension by a factor 3 , but surface tension forces vary by a factor of about $10^{4}$ for bubbles in the centimeter as compared to the meter range (equation (13)).

\section{Conclusions}

[60] We investigate the interface dynamics of a segregated gas phase in the context of basaltic volcanism. The two physical parameters of gas/basalt systems that may vary by several orders of magnitude are bubble size and magma viscosity. This variability in physical parameters translates into a wide range of relevant nondimensional regimes. The two most important nondimensional numbers to consider are $R e$ and $B o$, based on which we identify three fluid dynamical regimes (Figure 1). Resolving the detailed bubble dynamics is not equally important in each of the three scaling regimes. In fact, the bubble interactions may typically be neglected in the first regime. However, once bubbles become deformable $(B o>1)$ the fluid dynamical interactions between the bubbles begin to play an important role. The observation that deformability enhances coalescence has been investigated in detail in previous studies [Manga and Stone, 1993, 1994, 1995]. An important contribution of our study is the observation that deformability not only enhances coalescence but also breakup (section 5.2). At finite $R e$, we observe a balance of breakup and coalescence in our computations, with coalescence dominating at low $R e$ and breakup at large $R e$.

[61] Our simulations demonstrate that both bubbles and slugs are prone to dynamic instabilities, even in the absence of interactions with other bubbles/slugs. We observe two modes of breakup, gradual and catastrophic. A typical breakup sequence entails a small Rayleigh-Taylor instability forming at the leading surface of the bubble or slug. Surface tension is insufficient to restore the surface if the radius of the gas pocket exceeds $a_{c r}$ (equation (16)). Viscous forces, however, tend to stabilize the gas pocket through a combination of damping and lateral transport of the perturbation toward the periphery of the gas volume. The onset of breakup depends on the relative dominance of these processes (section 3). Interestingly, we find that while bubbles and slugs begin to break apart gradually at approximately the same $R e$, catastrophic breakup is observed at lower $R e$ for slugs than for bubbles. We suggest that the difference is partly due to the lower rise speed of large slugs as compared to bubbles of the same size and partly due to the more intense shearing of the slug surface near the conduit wall, which may give rise to KelvinHelmholtz instabilities.

[62] The breakup criteria provided in this paper are inevitably underestimates, for the following two reasons: First, all of our computations are based on spherical initial conditions, which are dynamically the most stable. However, bubbles or slugs with radii representative of the third scaling regime have probably formed through coalescence of smaller bubbles or breakup of an even larger bubble and will generally not be spherical. The fact that we commonly observe complex sequences of coalescence and breakup (Figure 9) in the third scaling regime attests to the additional complexity introduced by distorted bubble shapes. We also note that we observe dynamic instabilities even in the Stokes regime if the initial condition of the bubble or slug is not spherical. Second, we only discuss breakup in a stagnant fluid. Any additional complexities in the flow field such as shearing or turbulence will tend to aid breakup, because they facilitate the formation of destabilizing surface distortions [Clift et al., 2005]. For Stromboli in particular the existence of vigorous and possibly turbulent flow is not merely hypothetical [Misiti et al., 2009; Bertagnini et al., 2003].

[63] Applied to Stromboli, our simulations suggest that the presence of large stable gas slugs in the conduit requires zero or very low Re independent of whether the RSD or the CF model is invoked to explain slug formation (section 5). Since gas slugs at Stromboli are thought to represent gas volumes of several meters in size, unusually high viscosities (i.e., well above $O\left(10^{4}\right) \mathrm{Pa}$ s) would be needed to stabilize the slugs. While viscosities in this range are certainly possible [Misiti et al., 2009], geophysical data indicates that Strombolian slugs are characterized by rapid rise speeds $(10-70 \mathrm{~m} / \mathrm{s})$ [Harris and Ripepe, 2007] and surface oscillations [Vergniolle and Brandeis, 1996; Vergniolle et al., 1996]. This evidence is difficult to reconcile with zero $R e$. The apparent conflict between dynamic instability and finite $R e$ flow in Stromboli's plumbing system might be resolved in various ways including (1) the gas slugs causing normal Strombolian activity might be significantly smaller than previously thought and characterized by $R e \ll 80$, (2) either the acoustic signal and/or the eruption itself is caused by a mechanism other than the viscous ascent and burst of a large gas slug, and (3) there might be a stabilizing mechanism not accounted for in our computations such as non-Newtonian rheology or surfactants.

[64] Acknowledgments. This work was supported by the Director, Office of Science, Computational and Technology Research, U.S. Department of Energy, under contract DE-AC02-05CH1 1231, the NSF grant on Continental Dynamics EAR-0409373, and an NSF Astronomy CAREER award to Linda T. Elkins-Tanton. Partial support for J.-C. Nave was provided through NSF grant DMS-0813648. We would like to acknowledge Taras Gerya, Peter van Keken, and Kathy Cashman for their helpful reviews of this manuscript. 


\section{References}

Batchelor, G. K. (1987), The stability of a large gas bubble rising through liquid, J. Fluid Mech., 184, 399-422, doi:10.1017/S0022112087002945.

Batchelor, G. K. (2000), An Introduction to Fluid Dynamics, Cambridge Univ. Press, Cambridge, U. K.

Bellman, R., and R. Pennington (1954), Effects of surface tension and viscosity on Taylor instability, Q. Appl. Math., 12(2), 151-162.

Bertagnini, A., M. Coltelli, P. Landi, M. Pompilio, and M. Rosi (1999), Violent explosions yield new insights into dynamics of Stromboli volcano, Eos Trans. AGU, 80(52), 633, doi:10.1029/99EO00415.

Bertagnini, A., N. Métrich, P. Landi, and M. Rosi (2003), Stromboli volcano (Aeolian Archipelago, Italy): An open window on the deep-feeding system of a steady state basaltic volcano, J. Geophys. Res., 108(B7), 2336, doi:10.1029/2002JB002146.

Blackburn, E. A., L. Wilson, and R. S. J. Sparks (1976), Mechanisms and dynamics of Strombolian activity, J. Geol. Soc. London, 132, 429-440, doi:10.1144/gsjgs.132.4.0429.

Bretherton, F. (1961), The motion of long bubbles in tubes, J. Fluid Mech. 10(02), 166-188, doi:10.1017/S0022112061000160.

Capaldi, G., et al. (1978), Stromboli and its 1975 eruption, Bull. Volcanol., 41(3), 259-285, doi:10.1007/BF02597227.

Chorin, A. (1968), Numerical solution of the Navier-Stokes equations, Math. Comput., 22(104), 745-762, doi:10.2307/2004575.

Clift, R., J. R. Grace, and M. E. Weber (1974), Stability of bubbles in fluidized beds, Ind. Eng. Chem. Fundam., 13(1), 45-51, doi:10.1021/ i160049a009.

Clift, R., J. R. Grace, and M. E. Weber (2005), Bubbles, Drop, and Particles, Dover, New York.

Crowdy, D. G. (2003), Compressible bubbles in Stokes flow, J. Fluid Mech., 476, 345-356, doi:10.1017/S0022112002002975.

Davis, R. H. (1999), Buoyancy-driven viscous interaction of a rising drop with a smaller trailing drop, Phys. Fluids, 11(5), 1016-1028, doi:10.1063/1.869973.

Francalanci, L., P. Manetti, and A. Peccerillo (1989), Volcanological and magmatological evolution of Stromboli volcano (Aeolian Islands): The roles of fractional crystallization, magma mixing, crustal contamination and source heterogeneity, Bull. Volcanol., 51(5), 355-378, doi:10.1007/ BF01056897.

Francalanci, L., S. Tommasini, S. Conticelli, and G. R. Davies (1999) $\mathrm{Sr}$ isotope evidence for short magma residence time for the 20th century activity at Stromboli volcano, Italy, Earth Planet. Sci. Lett., 167(1-2), 61-69, doi:10.1016/S0012-821X(99)00013-8

Gardner, J. E., M. Hilton, and M. R. Carroll (1999), Experimental constraints on degassing of magma: isothermal bubble growth during continuous decompression from high pressure, Earth Planet. Sci. Lett., 168(1-2), 201-218, doi:10.1016/S0012-821X(99)00051-5.

Gonnermann, H. M., and M. Manga (2007), The fluid mechanics inside volcano, Annu. Rev. Fluid Mech., 39, 321-356, doi:10.1146/annurev. fluid.39.050905.110207

Grace, J. R., T. Wairegi, and T. H. Nguyen (1976), Shapes and velocities of single drops and bubbles moving freely through immiscible liquids, Trans. Inst. Chem. Eng., 54(3), 167-173.

Grace, J. R., T. Wairegi, and J. Brophy (1978), Break-up of drops and bubbles in stagnant media, Can. J. Chem. Eng., 56(1), 3-8, doi:10.1002/ cjce. 5450560101

Harris, A., and M. Ripepe (2007), Synergy of multiple geophysical approaches to unravel explosive eruption conduit and source dynamicsA case study from Stromboli, Chem. Erde, 67(1), 1-35, doi:10.1016/ j.chemer.2007.01.003.

Head, J. W., and L. Wilson (2003), Deep submarine pyroclastic eruptions: Theory and predicted landforms and deposits, J. Volcanol. Geotherm. Res., 121(3-4), 155-193, doi:10.1016/S0377-0273(02)00425-0.

Hurwitz, S., and O. Navon (1994), Bubble nucleation in rhyolitic melts: Experiments at high pressure, temperature, and water content, Earth Planet. Sci. Lett., 122(3-4), 267-280, doi:10.1016/0012-821X(94) 90001-9

Jaupart, C., and S. Vergniolle (1988), Laboratory models of Hawaiian and Strombolian eruptions, Nature, 331(6151), 58-60, doi:10.1038/ $331058 \mathrm{a} 0$.

Jaupart, C., and S. Vergniolle (1989), The generation and collapse of a foam layer at the roof of a basaltic magma chamber, J. Fluid Mech. 203, 347-380, doi:10.1017/S0022112089001497.

Kang, M., R. P. Fedkiw, and X. D. Liu (2000), A boundary condition capturing method for multiphase incompressible flow, J. Sci. Comput., 15(3), 323-360, doi:10.1023/A:1011178417620.

Klug, C., K. Cashman, and C. Bacon (2002), Structure and physical characteristics of pumice from the climactic eruption of Mount Mazama (Crater Lake), Oregon, Bull. Volcanol., 64(7), 486-501, doi:10.1007/s00445-002 0230-5.
Koh, C. J., and L. G. Leal (1989), The stability of drop shapes for translation at zero Reynolds number through a quiescent fluid, Phys. Fluids A, 1(8), 1309-1313, doi:10.1063/1.857359.

Kojima, M., E. J. Hinch, and A. Acrivos (1984), The formation and expansion of a toroidal drop moving in a viscous fluid, Phys. Fluids, 27(1), 19-32, doi:10.1063/1.864511.

Kushner, J., M. A. Rother, and R. H. Davis (2001), Buoyancy-driven interactions of viscous drops with deforming interfaces, J. Fluid Mech., 446, 253-269.

Liu, X.-D., R. P. Fedkiw, and M. Kang (2000), A boundary condition capturing method for Poisson's equation on irregular domains, J. Comput. Phys., 160(1), 151-178, doi:10.1006/jcph.2000.6444.

Lucas, D., E. Krepper, and H. Prasser (2005), Development of co-current air-water flow in a vertical pipe, Int. J. Multiphase Flow, 31(12), 1304-1328, doi:10.1016/j.ijmultiphaseflow.2005.07.004.

Machu, G., W. Meile, L. C. Nitsche, and U. Schaflinger (2001), Coalescence, torus formation and breakup of sedimenting drops: Experiments and computer simulations, J. Fluid Mech., 447, 299-336, doi:10.1017/ S0022112001005882.

Manga, M., and H. A. Stone (1993), Buoyancy-driven interactions between two deformable viscous drops, J. Fluid Mech., 256, 647-683, doi:10.1017/S0022112093002915.

Manga, M., and H. A. Stone (1994), Interactions between bubbles in magmas and lavas: Effects of bubble deformation, J. Volcanol. Geotherm. Res., 63, 267-279, doi:10.1016/0377-0273(94)90079-5.

Manga, M., and H. A. Stone (1995), Collective hydrodynamics of deformable drops and bubbles in dilute low Reynolds number suspensions, J. Fluid Mech., 300, 231-263, doi:10.1017/S0022112095003673.

Mangan, M. T., and K. V. Cashman (1996), The structure of basaltic scoria and reticulite and inferences for vesiculation, foam formation, and fragmentation in lava fountains, J. Volcanol. Geotherm. Res., 73(1-2), 1-18, doi:10.1016/0377-0273(96)00018-2.

Mangan, M. T., K. V. Cashman, and S. Newman (1993), Vesiculation of basaltic magma during eruption, Geology, 21(2), 157-160, doi:10.1130/ 0091-7613(1993)021<0157:VOBMDE $>2.3 . \mathrm{CO} ; 2$.

Menand, T., and J. C. Phillips (2007), Gas segregation in dykes and sills, J. Volcanol. Geotherm. Res., 159(4), 393-408, doi:10.1016/j.jvolgeores. 2006.08.003.

Misiti, V., F. Vetere, A. Mangiacapra, H. Behrens, A. Cavallo, P. Scarlato, and D. Dingwell (2009), Viscosity of high-K basalt from the 5th April 2003 Stromboli paroxysmal explosion, Chem. Geol., 260(3-4), 278-285, doi:10.1016/j.chemgeo.2008.12.023.

Mourtada-Bonnefoi, C. C., and D. Laporte (2002), Homogeneous bubble nucleation in rhyolitic magmas: An experimental study of the effect of $\mathrm{H}_{2} \mathrm{O}$ and $\mathrm{CO}_{2}$, J. Geophys. Res., 107(B4), 2066, doi:10.1029/ 2001JB000290.

Mudde, R. F. (2005), Gravity-driven bubbly flows, Annu. Rev. Fluid Mech., 37, 393-423, doi:10.1146/annurev.fluid.37.061903.175803.

Namiki, A., and M. Manga (2008), Transition between fragmentation and permeable outgassing of low viscosity magmas, J. Volcanol. Geotherm. Res., 169(1-2), 48-60, doi:10.1016/j.jvolgeores.2007.07.020.

Papale, P. (1999), Strain-induced magma fragmentation in explosive eruptions, Nature, 397(6718), 425-428, doi:10.1038/17109.

Parfitt, E. A., and L. Wilson (1995), Explosive volcanic eruptions-IX. The transition between Hawaiian-style lava fountaining and Strombolian explosive activity, Geophys. J. Int., 121(1), 226-232, doi:10.1111/ j.1365-246X.1995.tb03523.x.

Plesset, M., and C. Whipple (1974), Viscous effects in Rayleigh-Taylor instability, Phys. Fluids, 17(1), 1-7, doi:10.1063/1.1694570.

Pozrikidis, C. (1990), The instability of a moving viscous drop, J. Fluid Mech., 210, 1-21, doi:10.1017/S0022112090001203.

Pozrikidis, C. (2001), Expansion of a compressible gas bubble in Stokes flow, J. Fluid Mech., 442, 171-189, doi:10.1017/S0022112001004992.

Proussevitch, A. A., and D. L. Sahagian (1996), Dynamics of coupled diffusive and decompressive bubble growth in magmatic systems, J. Geophys. Res., 101(B8), 17,447-17,455, doi:10.1029/96JB01342.

Proussevitch, A., and D. Sahagian (2005), Bubbledrive-1: A numerical model of volcanic eruption mechanisms driven by disequilibrium magma degassing, J. Volcanol. Geotherm. Res., 143(1-3), 89-111, doi:10.1016/ j.jvolgeores.2004.09.012.

Ripepe, M., and E. Marchetti (2002), Array tracking of infrasonic sources at Stromboli volcano, Geophys. Res. Lett., 29(22), 2076, doi:10.1029/ 2002GL015452.

Rosi, M., A. Bertagnini, and P. Landi (2000), Onset of the persistent activity at Stromboli Volcano (Italy), Bull. Volcanol., 62(4-5), 294-300, doi:10.1007/s004450000098.

Rother, M. A., and R. H. Davis (2001), The effect of slight deformation on droplet coalescence in linear flows, Phys. Fluids, 13(5), 1178-1190, doi:10.1063/1.1358871 
Rother, M. A., and R. H. Davis (2005), Breakup and capture of two sedimenting drops in a vertical temperature gradient, Phys. Fluids, 17(3), 032103, doi:10.1063/1.1856532.

Rother, M. A., A. Z. Zinchenko, and R. H. Davis (1997), Buoyancy-driven coalescence of slightly deformable drops, J. Fluid Mech., 346, 117-148, doi:10.1017/S002211209700623X.

Ryerson, F. J., H. C. Weed, and A. J. Piwinskii (1988), Rheology of subliquidus magmas: 1. Picritic compositions, J. Geophys. Res., 93(B4), 3421-3436, doi:10.1029/JB093iB04p03421.

Shaw, H. R. (1969), Rheology of Basalt in the Melting Range, J. Petrol., 10(3), 510-535

Sparks, R. S. J. (1978), The dynamics of bubble formation and growth in magmas: A review and analysis, J. Volcanol. Geotherm. Res., 3(1-2), 1-37, doi:10.1016/0377-0273(78)90002-1.

Sparks, R. S. J., J. Barclay, C. Jaupart, H. M. Mader, and J. C. Phillips (1994), Physical aspects of magma degassing; 1, Experimental and theoretical constraints on vesiculation, in Volatiles in Magmas, Rev. Mineral. Geochem., vol. 30, edited by M. R. Carroll and J. R. Holloway, pp. 413-445, Mineral. Soc. of Am., Washington, D. C.

Spieler, O., B. Kennedy, U. Kueppers, D. B. Dingwell, B. Scheu, and J. Taddeucci (2004), The fragmentation threshold of pyroclastic rocks, Earth Planet. Sci. Lett., 226(1-2), 139-148, doi:10.1016/j.eps1. 2004.07.016.

Suckale, J., J.-C. Nave, and B. H. Hager (2010), It takes three to tango: 1. Simulating buoyancy-driven flow in the presence of large viscosity contrasts, J. Geophys. Res., 115, B07410, doi:10.1029/2009JB006916.

Toramaru, A. (1995), Numerical study of nucleation and growth of bubbles in viscous magmas, J. Geophys. Res., 100(B2), 1913-1931, doi:10.1029/ 94JB02775.

Vergniolle, S., and G. Brandeis (1994), Origin of the sound generated by Strombolian explosions, Geophys. Res. Lett., 21(18), 1959-1962, doi:10.1029/94GL01286.
Vergniolle, S., and G. Brandeis (1996), Strombolian explosions: 1. A large bubble breaking at the surface of a lava column as a source of sound, J. Geophys. Res., 101(B9), 20,433-20,447, doi:10.1029/96JB01178.

Vergniolle, S., and C. Jaupart (1986), Separated two-phase flow and basaltic eruptions, J. Geophys. Res., 91(B12), 12,842-12,860, doi:10.1029/ JB091iB12p12842.

Vergniolle, S., G. Brandeis, and J. C. Mareschal (1996), Strombolian explosions: 2. Eruption dynamics determined from acoustic measurements, J. Geophys. Res., 101(B9), 20,449-20,466, doi:10.1029/96JB01925.

Wegener, P. P., and J. Y. Parlange (1973), Spherical-cap bubbles, Annu. Rev. Fluid Mech., 5, 79-100, doi:10.1146/annurev.fl.05.010173.000455.

Wilson, L. (1980), Relationships between pressure, volatile content and ejecta velocity in three types of volcanic explosion, J. Volcanol. Geotherm. Res., 8(2-4), 297-313, doi:10.1016/0377-0273(80)90110-9.

Wilson, L., and J. W. Head (1981), Ascent and eruption of basaltic magma on the Earth and Moon, J. Geophys. Res., 86(B4), 2971-3001, doi:10.1029/JB086iB04p02971.

Zhang, Y. X. (1999), A criterion for the fragmentation of bubbly magma based on brittle failure theory, Nature, 402(6762), 648-650, doi:10.1038/45210.

Zinchenko, A. Z., M. A. Rother, and R. H. Davis (1997), A novel boundaryintegral algorithm for viscous interaction of deformable drops, Phys. Fluids, 9(6), 1493-1511, doi:10.1063/1.869275.

Zinchenko, A. Z., M. A. Rother, and R. H. Davis (1999), Cusping, capture, and breakup of interacting drops by a curvatureless boundary-integral algorithm, J. Fluid Mech., 391, 249-292, doi:10.1017/S0022112099005285.

L. T. Elkins-Tanton, B. H. Hager, and J. Suckale, Department of Earth, Atmospheric, and Planetary Sciences, Massachusetts Institute of Technology, 77 Massachusetts Ave, Cambridge, MA 02139, USA. (suckale@mit.edu)

J.-C. Nave, Department of Mathematics, Massachusetts Institute of Technology, 77 Massachusetts Ave, Cambridge, MA 02139, USA. 